TRANSACTIONS OF THE

AMERICAN MATHEMATICAL SOCIETY

Volume 356, Number 8, Pages 3077-3107

S 0002-9947(04)03517-2

Article electronically published on February 4, 2004

\title{
HOMOTOPY EQUIVALENCE OF TWO FAMILIES OF COMPLEXES
}

\author{
GIANDOMENICO BOFFI AND DAVID A. BUCHSBAUM
}

\begin{abstract}
An explicit homotopy equivalence is established between two families of complexes, both of which generalize the classical Koszul complex.
\end{abstract}

\section{Introduction}

Many years ago, in 2, a family of complexes was introduced which were to be considered generalizations of the usual Koszul complex, and in [3] they were studied in some detail, especially in relation to the generalized Cohen-Macaulay Theorem and a generalized multiplicity notion (see $[8$ and $[9]$ ). Given a commutative ring $R$, the idea was to take a map $f: R^{m} \rightarrow R^{n}(m \geq n)$ and, for each integer $k$, with $1 \leq k \leq n$, to associate a complex related to the map $\Lambda^{k} f: \Lambda^{k} R^{m} \rightarrow \Lambda^{k} R^{n}$ (we will denote it by $\mathbf{C}(k ; f)$ in this article). At about the same time, another - and much more efficient - complex was developed by Eagon and Northcott [6] which was associated to the map $\Lambda^{n} f$. A number of years later, Eisenbud and one of the authors $([5])$ constructed a large family of complexes which were associated to the maps $L_{\left(k, 1^{q}\right)}(f): L_{\left(k, 1^{q}\right)} R^{m} \rightarrow L_{\left(k, 1^{q}\right)} R^{n}$ induced on these hooks from the map $f$. In particular, for $q=0$, complexes were associated to $\Lambda^{k} f$ for all $1 \leq k \leq n$ (which we will denote by $\mathbf{T}(k ; f)$ in this article), and for $k=n$, the Eagon-Northcott complex mentioned above was reobtained. As was the case of the Eagon-Northcott complex, the complexes in [5] were much slimmer than the corresponding complexes constructed earlier in 2.1. Although a connection between these complexes was never doubted, none of the authors involved in the construction of these complexes ever established a connection between the more unwieldy - or fatter - ones and the slimmer ones (except for the case $k=n$ which was treated, albeit in a very awkward way, in [4]).

Another gap in the literature has to do with the fact that in [2] it was stated that for every maximal minor $\mu$ of $f$, the complex $\mathbf{C}(n ; f)$ carried a homotopy which made multiplication by $\mu$ homotopic to zero. In [3] an 'acyclicity-type' proof of the grade-sensitivity of the complexes $\mathbf{C}(k ; f)$ was given, which did not involve the use of homotopies, so that the cited homotopy was never written down in either of those papers, and no such homotopy was ever given for these complexes (although explicit use of it was made in [7]).

Received by the editors January 15, 2003.

2000 Mathematics Subject Classification. Primary 13D25.

${ }^{1}$ This explains the notation $\mathbf{C}(k ; f)$ and $\mathbf{T}(k ; f)$ : the $\mathbf{C}$ stands for 'corpulent', while the $\mathbf{T}$ stands for 'thin'. 
In this paper, we propose to fill in some of those gaps. In Section 2, we will show that for each maximal minor $\mu$ of the map $f$ there is a homotopy on $\mathbf{C}(k ; f)$ establishing that multiplication by $\mu$ is homotopic to zero (we will occasionally refer to this as the 'homothety homotopy'). In Section 3, we will show the existence of maps $\alpha: \mathbf{T}(k ; f) \rightarrow \mathbf{C}(k ; f)$ and $\theta: \mathbf{C}(k ; f) \rightarrow \mathbf{T}(k ; f)$ such that $\theta \alpha$ is the identity, that is, we will show that the complex $\mathbf{T}(k ; f)$ is a summand of $\mathbf{C}(k ; f)$. Using this fact, we will show that the homothety homotopy on the latter complex can be transported to its summand.

Finally, in Section 4 we will show that the composition $\alpha \theta$ is homotopic to the identity. Thus, the fact that the complexes $\mathbf{T}(k ; f)$ and $\mathbf{C}(k ; f)$ are homotopically equivalent will finally be established.

Throughout the paper, we will use notation that has become more or less standard in this context. We will let $F=R^{m}$ and $G=R^{n}$ and note that $\Lambda G^{*}$ operates on $\Lambda F$ (and $\Lambda F$ on $\Lambda G^{*}$ ) through $f^{*}$. For $\beta \in \Lambda G^{*}$ and $y \in \Lambda F$, we denote the action of $\beta$ on $y$ by $\beta(y)$. If we diagonalize an element $x$, we will generally write its diagonalization as $\sum x_{i} \otimes x_{i}^{\prime}$. If $x$ has degree $d$, and we want to specify its diagonalization in a fixed bidegree $(l, d-l)$, we will often write this diagonalization as $\sum x_{i l} \otimes x_{i d-l}^{\prime}$

\section{The 'Homothety homotopy'}

In this section, we define the homotopy on the complex $\mathbf{C}(k ; f)$. This complex is defined as follows:

$$
\begin{aligned}
0 \rightarrow C_{m-n+1}^{k} \rightarrow \cdots \rightarrow C_{q}^{k} \rightarrow \cdots & \rightarrow \sum_{s_{i} \geq 1} \Lambda^{n-k+s_{0}} G^{*} \otimes \Lambda^{s_{1}} G^{*} \otimes \Lambda^{n+|s|} F \\
& \rightarrow \sum_{s \geq 1} \Lambda^{n-k+s} G^{*} \otimes \Lambda^{n+s} F \rightarrow \Lambda^{k} F \rightarrow \Lambda^{k} G,
\end{aligned}
$$

where

$$
C_{q}^{k}=\sum_{s_{i} \geq 1} \Lambda^{n-k+s_{0}} G^{*} \otimes \Lambda^{s_{1}} G^{*} \otimes \cdots \otimes \Lambda^{s_{q-2}} G^{*} \otimes \Lambda^{n+|s|} F, \quad q \geq 2,
$$

$|s|=\sum s_{i}$, and the maps (except for $\Lambda^{k} f: \Lambda^{k} F \rightarrow \Lambda^{k} G$ ) are the bar complex maps associated to the action of the algebra $\Lambda G^{*}$ on $\Lambda F$.

As the signs of all our maps will be very crucial in all that we are about to do, we will make clear just what we mean by 'boundary map' in this context. Namely, if $a_{0} \otimes a_{1} \otimes \cdots \otimes a_{q-2} \otimes x \in C_{q}^{k}$ for $q \geq 3$, then

$$
\begin{aligned}
& \partial\left(a_{0} \otimes a_{1} \otimes \cdots \otimes a_{q-2} \otimes x\right) \\
= & a_{0} \otimes a_{1} \otimes \cdots \otimes a_{q-2}(x) \\
& +\sum_{i=0}^{q-3}(-1)^{q-i} a_{0} \otimes a_{1} \otimes \cdots \otimes a_{i} \wedge a_{i+1} \otimes \cdots \otimes a_{q-2} \otimes x .
\end{aligned}
$$

We take $\xi \in \Lambda^{n} G^{*}$ and $\lambda \in \Lambda^{n} F$, and we want to show that multiplication in the fat complex $\mathbf{C}(k ; f)$ by $\mu=\xi(\lambda)$ is homotopic to zero. We define

$$
\sigma_{0}: \Lambda^{k} G \rightarrow \Lambda^{k} F
$$

\footnotetext{
${ }^{2}$ Much of this could be handled more elegantly using letter-place notation. However, since our use of this notation is very limited in this paper, we will avoid introducing the more elaborate letter-place machinery.
} 
by setting

$$
\sigma_{0}(y)=y(\xi)(\lambda)
$$

Then we have

$$
\partial \sigma_{0}(y)=\sum \pm y_{i} \wedge \xi\left(y_{i}^{\prime} \wedge \lambda\right)=y \wedge \xi(\lambda)=\mu y .
$$

Most of the summands disappear in the above sum since the $\lambda$ is now to be considered as sitting inside $\Lambda^{n} G$, so that multiplication with $y_{i}^{\prime}$ is zero unless the degree of $y_{i}^{\prime}$ is zero. The first equality of (2.2) is a variant of the 'measuring formula' [5], which says that, for $\beta \in \Lambda^{i}\left(G^{*}\right)$ and $x, y \in \Lambda F$,

$$
\beta(x \wedge y)=\sum_{l=0}^{i} \pm \beta_{l}(x) \wedge \beta_{i-l}^{\prime}(y) .
$$

The signs \pm in this formula are explicitly given in [5].

We now define

$$
\sigma_{1}(x)=\sum_{l<k} \sum_{j} x_{j, l}(\xi) \otimes x_{j, k-l}^{\prime} \wedge \lambda,
$$

where $x \in \Lambda^{k} F$. Remember that the $l$ is supposed to indicate the degree of the term, and the $j$ indicates the summation.

In order to see that this works, we prove the following two lemmas:

Lemma 2.1. For $x \in \Lambda^{k} F, \xi$ and $\lambda$ as before, and for each integer $l$, we have

$$
\sum_{j} x_{j, l}(\xi)\left(x_{j, k-l}^{\prime} \wedge \lambda\right)=\sum_{j} \sum_{t=0}^{k-l}(-1)^{t}\left(\begin{array}{c}
l+t \\
l
\end{array}\right) x_{j, k-l-t}^{\prime} \wedge x_{j, l+t}(\xi)(\lambda) .
$$

Proof. By the measuring formula, we see that

$$
\begin{aligned}
\sum_{j} x_{j, l}(\xi)\left(x_{j, k-l}^{\prime} \wedge \lambda\right) & =\sum_{j} \sum_{i, t} \pm \xi_{i, t}\left(x_{j, k-l}^{\prime}\right) \wedge x_{j, l}\left(\xi_{i, n-t}\right)(\lambda) \\
& =\sum_{j} \sum_{i, t} \pm x_{j, k-l-t}^{\prime} \wedge x_{j, t}^{\prime \prime}\left(\xi_{i, t}\right) x_{j, l}\left(\xi_{i, n-t}\right)(\lambda) \\
& =\sum_{j} \sum_{t} \pm x_{j, k-l-t}^{\prime} \wedge x_{j, l}\left(\sum_{i} x_{j, t}^{\prime \prime}\left(\xi_{i, t}\right) \xi_{i, n-t}\right)(\lambda) \\
& =\sum_{j} \sum_{t} \pm x_{j, k-l-t}^{\prime} \wedge x_{j, l}\left(x_{j, t}^{\prime \prime}(\xi)\right)(\lambda) \\
& \left.=\sum_{j} \sum_{t} \pm\left(\begin{array}{c}
l+t \\
l
\end{array}\right) x_{j, k-l-t}^{\prime} \wedge x_{j, l+t}(\xi)\right)(\lambda) .
\end{aligned}
$$

Lemma 2.2. For $x \in \Lambda^{k} F$, and $\xi$ and $\lambda$ as before, we have

$$
\sum_{j} \sum_{l<k} x_{j, l}(\xi)\left(x_{j, k-l}^{\prime} \wedge \lambda\right)=x \wedge \xi(\lambda)-x(\xi) \wedge \lambda .
$$

Proof. As we saw above, for each $l$ we have

$$
x_{j, l}(\xi)\left(x_{j, k-l}^{\prime} \wedge \lambda\right)=\sum_{t=0}^{k-l}(-1)^{t}\left(\begin{array}{c}
l+t \\
l
\end{array}\right) x_{j, k-l-t}^{\prime} \wedge x_{j, l+t}(\xi)(\lambda) .
$$


So this says that

$$
\sum_{l<k} x_{j, l}(\xi)\left(x_{j, k-l}^{\prime} \wedge \lambda\right)=\sum_{\beta}(-1)^{\beta} \sum_{l}(-1)^{l}\left(\begin{array}{c}
\beta \\
l
\end{array}\right) x_{j, k-\beta}^{\prime} \wedge x_{j, \beta}(\xi)(\lambda),
$$

and the conclusion follows (because we know what happens to the alternating sum of binomial coefficients).

Now it is easy to see that $\sigma_{1}$ gives us what we need.

The next step is the 'generic' one; that is, once we get this one, the others all are of the same type. We define

$$
\sigma_{2}(\beta \otimes x)=\sum_{l<k-s} \sum_{j} \beta \otimes x_{j, n+s-k+l}(\xi) \otimes x_{j, k-l}^{\prime} \wedge \lambda
$$

for $\beta \in \Lambda^{n-k+s} G^{*}, x \in \Lambda^{n+s} F$. There are two 'tricks' to showing that this works: one is to recognize that

$$
\beta\left(x_{j, n+s-k+l}\right)(\xi)=\beta \wedge x_{j, n+s-k+l}(\xi)
$$

because $\xi$ is of degree $n$ (we are applying the same formula that we applied in the first equality of (2.2)). Then when we compute the boundary of $\sum_{l<k-s} \beta \otimes$ $x_{j, n+s-k+l}(\xi) \otimes x_{j, k-l}^{\prime} \wedge \lambda$, we can allow $l=k-s$, since the zero degree term in the middle cancels out in the boundary. But then, since the degree of $x$ is greater than $n$ (since $s \geq 1$ ), the term $x(\xi)=0$, so when we apply our lemma, we see that this definition of $s_{2}$ works.

Now it is easy to see that for $q \geq 2$, we may define

$\sigma_{q+1}\left(\beta_{0} \otimes \cdots \otimes \beta_{q-1} \otimes x\right)=\sum_{l<k-|s|} \sum_{j} \beta_{0} \otimes \cdots \otimes \beta_{q-1} \otimes x_{j, n+|s|-k+l}(\xi) \otimes x_{j, k-l}^{\prime} \wedge \lambda$

for $\beta_{0} \in \Lambda^{n-k+s_{0}} G^{*}, \beta_{i} \in \Lambda^{s_{i}} G^{*}$, for $i \geq 1$, and $x \in \Lambda^{n+|s|} F\left(|s|=\sum_{i \geq 0} s_{i}\right)$.

\section{THE MAPS $\alpha$ AND $\theta$}

The maps we want to define are between the complexes $\mathbf{C}(k ; f)$ and $\mathbf{T}(k ; f)$. The first complex has been described in the section above; we will define the complex $\mathbf{T}(k ; f)$ as

$$
\begin{aligned}
& 0 \rightarrow T_{m-n+1}^{k} \rightarrow \cdots \rightarrow T_{q}^{k} \rightarrow \cdots \rightarrow K_{\left(2,1^{n-k}\right)} G^{*} \otimes \Lambda^{n+2} F \\
& \rightarrow \Lambda^{n-k+1} G^{*} \otimes \Lambda^{n+1} F \rightarrow \Lambda^{k} F \rightarrow \Lambda^{k} G,
\end{aligned}
$$

where

$$
T_{q}^{k}=K_{\left(q-1,1^{n-k}\right)} G^{*} \otimes \Lambda^{n+q-1} F, \quad q \geq 2,
$$

and $K_{\left(q-1,1^{n-k}\right)} G^{*}$ denotes the Weyl module associated to the hook partition $\left(q-1,1^{n-k}\right)$. (Recall that the Weyl-or coSchur-module $K_{\left(l, 1^{m}\right)}$ is defined as the image of the map $\Lambda^{m} \otimes D_{l} \rightarrow \Lambda^{m+1} \otimes D_{l-1}$, where $D$ stands for the divided power. It is an instance of a more general module; cf. e.g. 11.) We should remark that $K_{\left(1,1^{n-k}\right)} G^{*}=\Lambda^{n-k+1} G^{*}$ (and $K_{\left(0,1^{n-k}\right)} G^{*}=0$ ). The map $\Lambda^{n-k+1} G^{*} \otimes \Lambda^{n+1} F \rightarrow \Lambda^{k} F$ is the usual action of the $\Lambda G^{*}$ on $\Lambda F$, and the other maps $K_{\left(q-1,1^{n-k}\right)} G^{*} \otimes \Lambda^{n+q-1} F \rightarrow K_{\left(q-2,1^{n-k}\right)} G^{*} \otimes \Lambda^{n+q-2} F$ are defined as follows: the modules $K_{\left(q-1,1^{n-k}\right)} G^{*} \otimes \Lambda^{n+q-1} F$ may be regarded as submodules of 
$\Lambda^{n-k+1} G^{*} \otimes D_{q-2} G^{*} \otimes \Lambda^{n+q-1} F$. By diagonalizing $D_{q-2} G^{*}$ into $D_{q-3} G^{*} \otimes D_{1} G^{*}$, and then acting by $G^{*}$ on $\Lambda F$, we see that we have a map

$$
\Lambda^{n-k+1} G^{*} \otimes D_{q-2} G^{*} \otimes \Lambda^{n+q-1} F \rightarrow \Lambda^{n-k+1} G^{*} \otimes D_{q-3} G^{*} \otimes \Lambda^{n+q-2} F .
$$

It is easy to see that this map, restricted to $T_{q}^{k}$, carries it into $T_{q-1}^{k}$ (see [5]).

3.1. The map $\alpha$. We may use the observation above to good advantage in defining the map $\alpha: \mathbf{T}(k ; f) \rightarrow \mathbf{C}(k ; f)$. In dimensions 0 and 1 , of course, the map is the identity. For ease of notation, we will label these maps $\alpha_{-2}$ and $\alpha_{-1}$, respectively, and, in general, we will denote by $\alpha_{q}$ the map that takes $T_{q+2}^{k}$ to $C_{q+2}^{k}$.

Definition of the map $\alpha$ : For $q=-2$ and -1 we define $\alpha_{q}$ to be the identity. For $q \geq 0$, we define $\alpha_{q}$ as the composition

$$
\begin{aligned}
T_{q+2}^{k} \hookrightarrow & \Lambda^{n-k+1} G^{*} \otimes D_{q} G^{*} \otimes \Lambda^{n+q+1} F \\
& \rightarrow \Lambda^{n-k+1} G^{*} \otimes \underbrace{D_{1} G^{*} \otimes \cdots \otimes D_{1} G^{*}}_{q} \otimes \Lambda^{n+q+1} F,
\end{aligned}
$$

where the right arrow is the $q$-fold diagonalization of $D_{q} G^{*}$. (We observe that the latter term is a summand of $C_{q+2}^{k}$.)

It is a relatively straightforward calculation to see that $\alpha$, thus defined, is a map of complexes.

3.2. The map $\theta$. The map $\theta: \mathbf{C}(k ; f) \rightarrow \mathbf{T}(k ; f)$ is a bit more complicated to define (except in dimensions 0 and 1 where, again, it is defined to be the identity and denoted by $\theta_{-2}$ and $\theta_{-1}$ ). As in the case of the definition of the map $\alpha$, we will denote by $\theta_{q}$ the map that sends $C_{q+2}^{k}$ to $T_{q+2}^{k}$. We introduce some notation to facilitate its definition.

Notation: Assume that a fixed basis of $G^{*}$ is given, say $y_{1}, \ldots, y_{n}$. An element $y_{j_{1}} \wedge \cdots \wedge y_{j_{l}}$ will be written either as $j_{1} \wedge \cdots \wedge j_{l}$ or $j_{1} \cdots j_{l}$ or simply as $J$. In short, the index on a basis element will be used to denote that element (as is the practice when working with tableaux), and products of elements will be denoted by products of their indices. For $l=1$, we will usually write $j$ or $j_{1}$ instead of $J$. When working with products in the divided power algebra, we will use tableau notation in order to avoid confusion about whether juxtaposition means the usual product within that algebra, or the divided power when there are repeats. For example, we will write,

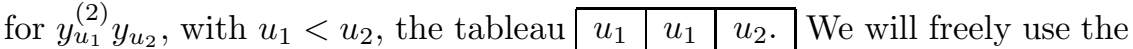
standard basis, consisting of standard tableaux, for Weyl modules, and a typical basis element of $T_{q+2}^{k}$ would be denoted by

\begin{tabular}{|l|l|l|l|}
\hline$j_{1}$ & $u_{1}$ & $\cdots$ & $u_{q}$ \\
\hline$j_{2}$ & \multicolumn{3}{|c}{} \\
\cline { 1 - 1 } & & \multicolumn{3}{|}{}
\end{tabular}$\otimes x$

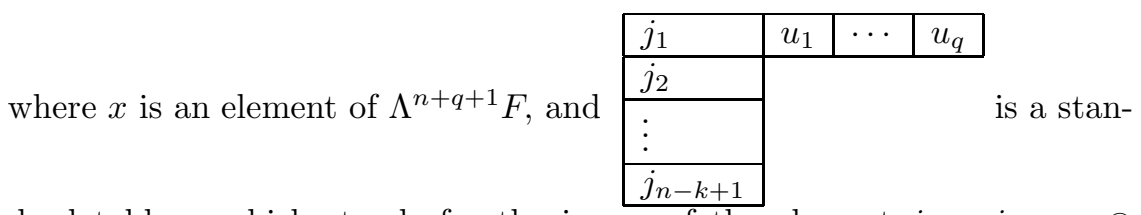
dard tableau which stands for the image of the element $j_{2} \cdots j_{n-k+1} \otimes$ 


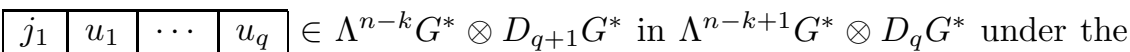
map which diagonalizes $D_{q+1} G^{*} \rightarrow G^{*} \otimes D_{q} G^{*}$, and then multiplies $\Lambda^{n-k} G^{*}$ into $\Lambda^{n-k+1} G^{*}$ by using the $G^{*}$ factor.

Recall that 'standard tableau' means that the indices are strictly increasing in the column, and weakly increasing in the row. For reasons that will become apparent later, we will make one more (unusual) convention about our use of tableau notation in the case of rows: we will assume that the tableau is zero if the top row is not weakly increasing as written. Thus, in the case of $y_{u_{1}}^{(2)} y_{u_{2}}$ with $u_{1}>u_{2}$, we would have to write \begin{tabular}{l|l|l|}
\hline$u_{2}$ & $u_{1}$ & $u_{1}$ \\
\hline
\end{tabular} to represent it as a tableau.

We are now in a position to define our maps $\theta_{q}$ for all $q \geq-2$.

Definition of the map $\theta$ : For $q=-2$ and -1 , we have already said that the map is to be the identity. For $q=0$, and $Y=J \otimes x$, with $J$ a basis element of $\Lambda^{n-k+s} G^{*}$ and $x \in \Lambda^{n+s} F$, we define

$$
\theta_{0}(Y)=(-1)^{(s-1)(n-k+1)} j_{s} \cdots j_{n-k+s} \otimes j_{1} \cdots j_{s-1}(x) .
$$

For $q>0$, and $Y=J \otimes u_{1} \otimes \cdots \otimes u_{q} \otimes x$ with all the $u_{i}$ basis elements of degree one, and $J$ still of degree $n-k+s$, we define

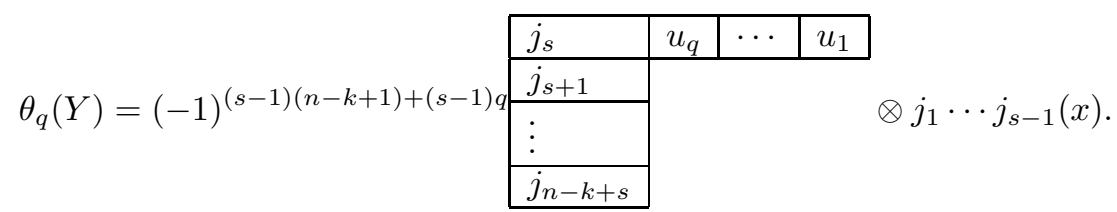

It is essential to remember here that the tableau is to be read as equal to zero if the row is not standard. Assume that the map $\theta_{l}$ has been defined on elements $Y^{\prime}=J \otimes U_{1} \otimes \cdots \otimes U_{l} \otimes x$ for $U_{i}$ basis elements of arbitrary degree, and $l<q$, and that $\theta_{q}$ has been defined on elements $Z=J \otimes U_{1} \otimes \cdots \otimes U_{q} \otimes x$ with $U_{1}, \ldots, U_{t}$ basis elements of arbitrary degree (we make the convention that $\left.U_{0}=J\right), U_{t+2}, \ldots, U_{q}$ basis elements of degree 1 , and $U_{t+1}$ basis element of degree $s_{t+1} \leq r(r>0)$. We now let $Z^{\prime}=J \otimes U_{1} \otimes \cdots \otimes U_{t+1} \otimes$ $\cdots \otimes U_{q} \otimes x$ with the basis element $U_{t+1}=v \wedge W, v$ of degree 1 , all of the basis elements $U_{t+2}, \ldots, U_{q}$ are of degree 1 , and degree $(W)=r$. Define

$$
\theta_{q}\left(Z^{\prime}\right)=\theta_{q}\left(B+(-1)^{(q-t-1) r} E\right),
$$

where $B=J \otimes U_{1} \otimes \cdots \otimes U_{t} v \otimes W \otimes U_{t+2} \otimes \cdots \otimes U_{q} \otimes x$, and $E=$ $J \otimes U_{1} \otimes \cdots \otimes U_{t} \otimes v \otimes U_{t+2} \otimes \cdots \otimes U_{q} \otimes W(x)$.

Notice that in position $t+1$, the elements $B$ and $E$ are of a degree less than or equal to $r$, while the terms of higher index are not affected in degree. From this we immediately see (using a simple induction proof) that $\theta_{q}\left(Z^{\prime}\right)=0$ unless $U_{t+2} \geq \cdots \geq U_{q}$.

While it is trivial to show that the map $\alpha$ is a map of complexes, it is not trivial to prove that the same is true of $\theta$. In order to prove this fact, we must first prove the following two lemmas.

Lemma 3.1. Let $A=J \otimes U_{1} \otimes \cdots \otimes U_{q} \otimes x$ be a 'basis' element of $\Lambda^{n-k+s_{0}} G^{*} \otimes$ $\Lambda^{s_{1}} G^{*} \otimes \cdots \otimes \Lambda^{s_{q}} G^{*} \otimes \Lambda^{n+|s|} F$, where by 'basis' element we mean that $J$ and all the $U_{i}$ are basis elements of their corresponding exterior powers (the element $x$ need not be a basis element). Fix an index $t$ with $0 \leq t \leq q-1$, and suppose that $U_{t+1}=v \wedge W$ with $v$ of degree 1 and $W$ of degree $r$, and that $s_{t+2}=s_{t+3}=\cdots=s_{q}=1$. We let 
$\rho=q-t-1$, we set $B=J \otimes U_{1} \otimes \cdots \otimes U_{t} v \otimes W \otimes U_{t+2} \otimes \cdots \otimes U_{q} \otimes x$ and set $E=J \otimes U_{1} \otimes \cdots \otimes U_{t} \otimes v \otimes U_{t+2} \otimes \cdots \otimes U_{q} \otimes W(x)$. Then

$$
\theta_{q-1} \partial_{C} A=\theta_{q-1} \partial_{C}\left\{B+(-1)^{\rho r} E\right\} .
$$

Proof. When $q=1$, this property is clearly satisfied, so we may assume that $q \geq 2$, and that this all holds for $q-1$. With this inductive assumption, the case $t=q-1$ is easy to prove, so we may assume that $t<q-1$. Since all the work in this lemma involves the application of $\theta_{q-1}$, we will denote it by $\theta$. The proof proceeds by meticulously calculating and keeping track of signs. We will use lowercase letters $u_{i}$ for $U_{i}$ when $s_{i}=1$.

$$
\begin{aligned}
\partial_{C} A= & J \otimes U_{1} \otimes \cdots \otimes U_{t} \otimes v W \otimes \partial\left(u_{t+2} \otimes \cdots \otimes u_{q} \otimes x\right) \\
& +(-1)^{q-t-1} J \otimes U_{1} \otimes \cdots \otimes U_{t} \otimes v W u_{t+2} \otimes \cdots \otimes u_{q} \otimes x \\
& +(-1)^{q-t} J \otimes U_{1} \otimes \cdots \otimes U_{t} v W \otimes u_{t+2} \otimes \cdots \otimes u_{q} \otimes x \\
& +(-1)^{q-t+1} \delta\left(J \otimes U_{1} \otimes \cdots \otimes U_{t} v\right) \otimes W \otimes u_{t+2} \otimes \cdots \otimes u_{q} \otimes x \\
& +(-1)^{q-t+1+\rho r} \delta\left(J \otimes U_{1} \otimes \cdots \otimes U_{t}\right) \otimes v \otimes u_{t+2} \otimes \cdots \otimes u_{q} \otimes W(x) .
\end{aligned}
$$

The use of the symbol $\partial$ has the meaning indicated by Formula (2.1), and involves the action of $\Lambda G^{*}$ on $\Lambda F$, while the symbol $\delta$ denotes the boundary of a bar complex in which the action of $\Lambda G^{*}$ on $\Lambda F$ does not enter. We are also using the convention of leaving out the $\theta$ in writing all the above (and following) equalities. For example, the last two lines above are really there by virtue of the definition of this map. Now we continue to compute

$$
\begin{aligned}
\partial_{C} B= & J \otimes U_{1} \otimes \cdots \otimes U_{t} v \otimes W \otimes \partial\left(u_{t+2} \otimes \cdots \otimes u_{q} \otimes x\right) \\
& +(-1)^{q-t-1} J \otimes U_{1} \otimes \cdots \otimes U_{t} v \otimes W u_{t+2} \otimes \cdots \otimes u_{q} \otimes x \\
& +(-1)^{q-t} J \otimes U_{1} \otimes \cdots \otimes U_{t} v W \otimes u_{t+2} \otimes \cdots \otimes u_{q} \otimes x \\
& +(-1)^{q-t+1} \delta\left(J \otimes U_{1} \otimes \cdots \otimes U_{t} v\right) \otimes W \otimes u_{t+2} \otimes \cdots \otimes u_{q} \otimes x
\end{aligned}
$$

and

$$
=(-1)^{\rho r}\left\{\begin{array}{c}
(-1)^{\rho r} \partial_{C} E \\
+(-1)^{q-t-1} J \otimes U_{1} \otimes \cdots \otimes U_{t} \otimes v u_{t+2} \otimes \cdots \otimes u_{q} \otimes W(x) \\
+(-1)^{q-t} J \otimes U_{1} \otimes \cdots \otimes U_{t} v \otimes u_{t+2} \otimes \cdots \otimes u_{q} \otimes W(x) \\
+(-1)^{q-t+1} \delta\left(J \otimes U_{1} \otimes \cdots \otimes U_{t}\right) \otimes v \otimes u_{t+2} \otimes \cdots \otimes u_{q} \otimes W(x)
\end{array}\right\} .
$$

Making obvious cancellations, then applying $\theta$ to the term $J \otimes U_{1} \otimes \cdots \otimes U_{t} \otimes v W \otimes$ $\partial\left(u_{t+2} \otimes \cdots \otimes u_{q} \otimes x\right)$ and splitting it up judiciously (as we will be doing to similar terms later), we get further cancellations, and we are left with having to prove the following:

$$
\begin{aligned}
& \theta\left(J \otimes U_{1} \otimes \cdots \otimes U_{t} \otimes v W u_{t+2} \otimes \cdots \otimes u_{q} \otimes x\right) \\
= & \theta\left(J \otimes U_{1} \otimes \cdots \otimes U_{t} v \otimes W u_{t+2} \otimes \cdots \otimes u_{q} \otimes x\right) \\
& +(-1)^{\rho r} \theta\left(J \otimes U_{1} \otimes \cdots \otimes U_{t} \otimes v u_{t+2} \otimes \cdots \otimes u_{q} \otimes W(x)\right) \\
& -(-1)^{\rho r} \theta\left(J \otimes U_{1} \otimes \cdots \otimes U_{t} v \otimes u_{t+2} \otimes \cdots \otimes u_{q} \otimes W(x)\right) .
\end{aligned}
$$

(These are simply the terms that are left after the above cancellations.)

Since our definition of $\theta$ requires that all our elements (except $x$ ) be basis elements, we have to 'rectify' our term $v W u_{t+2}$ in order to see what results when we apply $\theta$. First of all, we notice that if $u_{t+2}$ is equal to $v$, then it is trivial to prove 
the desired equality (because several of the terms drop out). If $v<u_{t+2}$, it is clear that

$$
\begin{aligned}
& \theta\left(J \otimes U_{1} \otimes \cdots \otimes U_{t} \otimes v W u_{t+2} \otimes \cdots \otimes u_{q} \otimes x\right) \\
= & \theta\left(J \otimes U_{1} \otimes \cdots \otimes U_{t} v \otimes W u_{t+2} \otimes \cdots \otimes u_{q} \otimes x\right) \\
& +(-1)^{c(r+1)} \theta\left(J \otimes U_{1} \otimes \cdots \otimes U_{t} \otimes v \otimes \cdots \otimes u_{q} \otimes W u_{t+2}(x)\right) .
\end{aligned}
$$

But we also have

$$
\begin{aligned}
& (-1)^{\rho r} \theta\left(J \otimes U_{1} \otimes \cdots \otimes U_{t} \otimes v u_{t+2} \otimes \cdots \otimes u_{q} \otimes W(x)\right) \\
= & (-1)^{\rho r} \theta\left(J \otimes U_{1} \otimes \cdots \otimes U_{t} v \otimes u_{t+2} \otimes \cdots \otimes u_{q} \otimes W(x)\right) \\
& +(-1)^{\rho r+c} \theta\left(J \otimes U_{1} \otimes \cdots \otimes U_{t} \otimes v \otimes \cdots \otimes u_{q} \otimes u_{t+2} W(x)\right),
\end{aligned}
$$

so we must show that

$$
\begin{aligned}
& (-1)^{c(r+1)} \theta\left(J \otimes U_{1} \otimes \cdots \otimes U_{t} \otimes v \otimes \cdots \otimes u_{q} \otimes W u_{t+2}(x)\right) \\
= & (-1)^{\rho r+c} \theta\left(J \otimes U_{1} \otimes \cdots \otimes U_{t} \otimes v \otimes \cdots \otimes u_{q} \otimes u_{t+2} W(x)\right) \\
= & (-1)^{\rho r+c+r} \theta\left(J \otimes U_{1} \otimes \cdots \otimes U_{t} \otimes v \otimes \cdots \otimes u_{q} \otimes W u_{t+2}(x)\right) .
\end{aligned}
$$

Noting that $\rho=c+1$, we see that $\rho r+c+r \equiv c(r+1) \bmod 2$, and our equality is true.

If $v>u_{t+2}$, we observe that $v W u_{t+2}=(-1)^{r+1} u_{t+2} v W$ and use the previous case together with some easy extra work.

Our second lemma that we need for our theorem is relatively straightforward.

Lemma 3.2. Let $A=J \otimes u_{1} \otimes \cdots \otimes u_{q} \otimes x$ be such that all the $u_{i}$ are of degree 1 . Then $\theta_{q-1} \partial_{C} A=0$ unless $u_{1} \geq u_{2} \geq \cdots \geq u_{q}$.

Proof. For $q=1$, there is nothing to prove. For $q=2$, we assume that $u_{1}<u_{2}$, and we see that

$$
\theta_{1} \partial_{C} A=\theta_{1}\left(J u_{1} \otimes u_{2} \otimes x-J \otimes u_{1} u_{2} \otimes x+J \otimes u_{1} \otimes u_{2}(x)\right) .
$$

But

$$
\theta_{1}\left(J u_{1} \otimes u_{2} \otimes x+J \otimes u_{1} \otimes u_{2}(x)\right)=\theta_{1}\left(J \otimes u_{1} u_{2} \otimes x\right)
$$

and we have it for $q=2$. To proceed to the general case, recall that if $B=$ $J \otimes u_{1} \otimes \cdots \otimes u_{q-1} \otimes x$, then $\theta_{q-1}(B)=0$ unless $u_{1} \geq u_{2} \geq \cdots \geq u_{q-1}$.

Assume, then, that we have $u_{l} \geq u_{l+1} \geq \cdots \geq u_{q}$, that $u_{l-1}<u_{l}$, and that $l<q$. Then

$$
\begin{aligned}
(-1)^{q} \partial_{C} A= & \sum_{r=0}^{l-3}(-1)^{r} J \otimes \cdots \otimes u_{r} u_{r+1} \otimes \cdots \otimes u_{l-1} \otimes u_{l} \otimes \cdots \otimes u_{q} \otimes x \\
& +(-1)^{l-2} J \otimes \cdots \otimes u_{l-2} u_{l-1} \otimes u_{l} \otimes \cdots \otimes u_{q} \otimes x \\
& +(-1)^{l-1} J \otimes \cdots \otimes u_{l-2} \otimes u_{l-1} u_{l} \otimes \cdots \otimes u_{q} \otimes x \\
& +(-1)^{l+1} J \otimes \cdots \otimes u_{l-1} \otimes u_{l+1} u_{l} \otimes \cdots \otimes u_{q} \otimes x \\
& +(-1)^{l} \sum_{r=1}^{q-l-1}(-1)^{r+1} J \otimes \cdots \otimes u_{l-1} \otimes \cdots \otimes u_{l+r+1} u_{l+r} \otimes \cdots \otimes u_{q} \otimes x \\
& +(-1)^{q} J \otimes u_{1} \otimes \cdots \otimes u_{q-1} \otimes u_{q}(x) .
\end{aligned}
$$

Now all of the terms $J \otimes \cdots \otimes u_{r} u_{r+1} \otimes \cdots \otimes u_{l-1} \otimes u_{l} \otimes \cdots \otimes u_{q} \otimes x$ go to zero under $\theta_{q-1}$ because our defining identities all take place to the left of $u_{l-1}$ and thus give 
us linear terms all containing $u_{l-1} \otimes u_{l}$ which, by the property of $\theta_{q-1}$, all go to zero. The reduction of the element in the third line cancels the term in the second line, and leaves the term

$$
\begin{aligned}
& (-1)^{l-1+q-l} J \otimes \cdots \otimes u_{l-2} \otimes u_{l-1} \otimes u_{l+1} \cdots \otimes u_{q} \otimes u_{l}(x) \\
= & (-1)^{q-1} J \otimes \cdots \otimes u_{l-2} \otimes u_{l-1} \otimes u_{l+1} \cdots \otimes u_{q} \otimes u_{l}(x) .
\end{aligned}
$$

The fourth line reduces to

$$
\begin{aligned}
& (-1)^{l+1} J \otimes \cdots \otimes u_{l-1} u_{l+1} \otimes u_{l} \otimes \cdots \otimes u_{q} \otimes x \\
& +(-1)^{l+1+q-l-1} J \otimes \cdots \otimes u_{l-1} \otimes u_{l+1} \otimes \cdots \otimes u_{q} \otimes u_{l}(x) .
\end{aligned}
$$

The second term has the opposite sign to the identical term above, so they cancel, and we are left with the term in the top line above. But $u_{l-1}$ and $u_{l+1}$ are both less than $u_{l}$, and so in the defining identities, the final linearization is going to contain either $u_{l-1}$ or $u_{l+1}$ to the left of $u_{l}$ and therefore is sent to zero by $\theta_{q-1}$. Thus we are left with the terms

$$
\begin{aligned}
& (-1)^{l} \sum_{r=1}^{q-l-1}(-1)^{r+1} J \otimes \cdots \otimes u_{l-1} \otimes u_{l} \otimes \cdots \otimes u_{l+r+1} u_{l+r} \otimes \cdots \otimes u_{q} \otimes x \\
& +(-1)^{q} J \otimes u_{1} \otimes \cdots \otimes u_{q-1} \otimes u_{q}(x) .
\end{aligned}
$$

The terms in the sum, because we assume the indices decrease beyond $l$, all reduce to linear terms containing $J \otimes \cdots \otimes u_{l-1} \otimes u_{l} \otimes \cdots$ which are sent by $\theta_{q-1}$ to zero. Reducing this term further gives us

$$
\begin{aligned}
& \pm J \otimes \cdots \otimes u_{l-1} u_{l+r+1} \otimes u_{l} \otimes u_{l+1} \otimes \cdots \otimes u_{l+r} \otimes \cdots \otimes u_{q} \otimes x \\
& \pm J \otimes \cdots \otimes u_{l-1} \otimes u_{l+r+1} \otimes u_{l+1} \otimes \cdots \otimes u_{l+r} \otimes \cdots \otimes u_{q} \otimes u_{l}(x) .
\end{aligned}
$$

Now both of these terms are sent to zero under $\theta_{q-1}$; the first one because further linearizations of the quadratic will reduce to linear terms having either $u_{l-1}$ or $u_{l+r+1}$ to the right of $u_{l}$, and the second one because, with $r \geq 1$, we have $u_{l+r+1}<$ $u_{l+1}$, and this violation of order gets it sent to zero.

The term $J \otimes u_{1} \otimes \cdots \otimes u_{q-1} \otimes u_{q}(x)$ gets sent to zero, since $l<q$.

We now consider the case $l=q$, which has been left out. In that event, the proof proceeds pretty much as above, with cancellation occurring among the very last terms, and the other terms equalling zero because of the occurrence of $u_{q-1} \otimes u_{q}$.

With these two lemmas, we are ready to prove the following theorem.

Theorem 3.3. The map $\theta$ defined above is a map of complexes. That is, if we let $\partial_{C}$ and $\partial_{T}$ be the boundary maps of $\mathbf{C}(k ; f)$ and $\mathbf{T}(k ; f)$, respectively, then we have

$$
\partial_{T} \theta_{q}=\theta_{q-1} \partial_{C}
$$

for all $q \geq-1$.

Proof. For $q=-1$, this is clear; the first place we must look is at $q=0$, i.e., we must see that $\partial_{T} \theta_{0}=\partial_{C}$. But

$$
\partial_{T} \theta_{0}(J \otimes x)=(-1)^{(s-1)(n-k+1)} j_{s} \cdots j_{n-k+s}\left(j_{1} \cdots j_{s-1}(x)\right)=J(x),
$$

and this last is clearly $\partial_{C}(J \otimes x)$.

To proceed to the general case, we use the two lemmas above; if we let $Y=$ $J \otimes U_{1} \otimes \cdots \otimes U_{q} \otimes x$, we observe that Lemmas 3.1 and 3.2 permit us to reduce to the case where all the $U_{i}$ are of degree one (hence we will use lowercase $u$ to denote 
them), and $u_{1} \geq \cdots \geq u_{q}$. In this case, we assume that $J$ is of degree $n-k+s$, and we must prove that

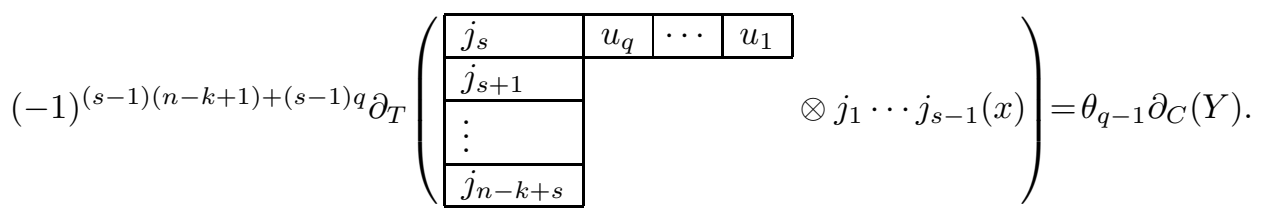

At this point, we must introduce some additional notation. To take account of the fact that not all the $u_{i}$ need be distinct, we will write

$$
Y=J \otimes u_{1}^{\otimes m_{1}} \otimes \cdots \otimes u_{p}^{\otimes m_{p}} \otimes x
$$

with $u_{1}>\cdots>u_{p}$.

Applying Lemma 3.1 (which we will refer to as the reduction formulas), as well as Lemma 3.2, it is straightforward to see that

$$
\begin{aligned}
\theta_{q-1} \partial_{C}(Y)= & (-1)^{q} \sum_{i=1}^{p} \theta_{q-1}\left(J u_{i} \otimes u_{1}^{\otimes m_{1}} \otimes \cdots \otimes u_{i}^{\otimes m_{i}-1} \otimes \cdots \otimes u_{p}^{\otimes m_{p}} \otimes x\right) \\
& +\sum_{i=1}^{p} \theta_{q-1}\left(J \otimes u_{1}^{\otimes m_{1}} \otimes \cdots \otimes u_{i}^{\otimes m_{i}-1} \otimes \cdots \otimes u_{p}^{\otimes m_{p}} \otimes u_{i}(x)\right) .
\end{aligned}
$$

To briefly sketch a proof of this fact, we first observe that

$$
\begin{aligned}
\partial_{C}(Y)= & \sum_{i=2}^{p}(-1)^{m_{p}+\cdots+m_{i}} J \otimes u_{1}^{\otimes m_{1}} \otimes \cdots \otimes u_{i-1}^{\otimes m_{i-1}-1} \\
& \otimes u_{i-1} u_{i} \otimes u_{i}^{\otimes m_{i}-1} \otimes \cdots \otimes u_{p}^{\otimes m_{p}} \otimes x \\
& +J \otimes u_{1}^{\otimes m_{1}} \otimes \cdots \otimes u_{p}^{\otimes m_{p}-1} \otimes u_{p}(x) \\
& +(-1)^{q} J u_{1} \otimes u_{1}^{\otimes m_{1}-1} \otimes \cdots \otimes u_{p}^{\otimes m_{p}} \otimes x
\end{aligned}
$$

and we want to evaluate $\theta_{q-1}$ on each of the terms in the sum for $i=2, \ldots, p$. Using our reduction formulas, as well as the facts that $u_{i}<u_{i-1}$ and $u_{i-1} u_{i}=-u_{i} u_{i-1}$, we have

$$
\begin{aligned}
& \theta_{q-1}\left(J \otimes u_{1}^{\otimes m_{1}} \otimes \cdots \otimes u_{i-1}^{\otimes m_{i-1}-1} \otimes u_{i-1} u_{i} \otimes u_{i}^{\otimes m_{i}-1} \otimes \cdots \otimes u_{p}^{\otimes m_{p}} \otimes x\right) \\
= & -\theta_{q-1}\left(J \otimes u_{1}^{\otimes m_{1}} \otimes \cdots \otimes u_{i-1}^{\otimes m_{i-1}-2} \otimes u_{i-1} u_{i} \otimes u_{i-1} \otimes u_{i}^{\otimes m_{i}-1} \otimes \cdots \otimes u_{p}^{\otimes m_{p}} \otimes x\right) \\
& +(-1)^{m_{p}+\cdots+m_{i}} \theta_{q-1}\left(J \otimes u_{1}^{\otimes m_{1}} \otimes \cdots \otimes u_{i-1}^{\otimes m_{i-1}-1} \otimes u_{i}^{\otimes m_{i}} \otimes \cdots \otimes u_{p}^{\otimes m_{p}} \otimes u_{i-1}(x)\right) .
\end{aligned}
$$

If we now apply the reduction formulas to the quadratic term

$-\theta_{q-1}\left(J \otimes u_{1}^{\otimes m_{1}} \otimes \cdots \otimes u_{i-1}^{\otimes m_{i-1}-2} \otimes u_{i-1} u_{i} \otimes u_{i-1} \otimes u_{i}^{\otimes m_{i}-1} \otimes \cdots \otimes u_{p}^{\otimes m_{p}} \otimes x\right)$,

we see that we get a new quadratic term plus a linear term that places $u_{i}$ to the left of $u_{i-1}$, which $\theta_{q-1}$ then carries to zero. Thus, each of these quadratic terms produces only one summand as we continue to apply the reduction formulas, and we may stop 'reducing' when we arrive at the point where $u_{i}$ multiplies $J$. Thus we 
see that

$$
\begin{aligned}
& \theta_{q-1}\left(J \otimes u_{1}^{\otimes m_{1}} \otimes \cdots \otimes u_{i-1}^{\otimes m_{i-1}-1} \otimes u_{i-1} u_{i} \otimes u_{i}^{\otimes m_{i}-1} \otimes \cdots \otimes u_{p}^{\otimes m_{p}} \otimes x\right) \\
= & (-1)^{m_{1}+\cdots+m_{i-1}} \theta_{q-1}\left(J u_{i} \otimes u_{1}^{\otimes m_{1}} \otimes \cdots \otimes u_{i}^{\otimes m_{i}-1} \otimes \cdots \otimes u_{p}^{\otimes m_{p}} \otimes x\right) \\
& +(-1)^{m_{p}+\cdots+m_{i}} \theta_{q-1}\left(J \otimes u_{1}^{\otimes m_{1}} \otimes \cdots \otimes u_{i-1}^{\otimes m_{i-1}-1} \otimes u_{i}^{\otimes m_{i}} \otimes \cdots \otimes u_{p}^{\otimes m_{p}} \otimes u_{i-1}(x)\right) .
\end{aligned}
$$

Multiplying each such term by $(-1)^{m_{p}+\cdots+m_{i}}$, gives us the claimed result.

Since, by our definition (and convention), $\theta_{q}(Y)=0$ unless $j_{s} \leq u_{p}$, we first have to show that $\theta_{q-1} \partial_{C}(Y)=0$ if $u_{p}<j_{s}$. In that case, it is clear that $\theta_{q-1}\left(J \otimes u_{1}^{\otimes m_{1}} \otimes \cdots \otimes u_{i}^{\otimes m_{i}-1} \otimes \cdots \otimes u_{p}^{\otimes m_{p}} \otimes u_{i}(x)\right)=0$ for all $i<p$, as is also $\theta_{q-1}\left(J u_{i} \otimes u_{1}^{\otimes m_{1}} \otimes \cdots \otimes u_{i}^{\otimes m_{i}-1} \otimes \cdots \otimes u_{p}^{\otimes m_{p}} \otimes x\right)$. The latter is true because if $u_{p}<j_{s}$, then the $(s+1)$ th factor in $J u_{i}$ is either $j_{s}$ or $u_{i}$. In either case (since $u_{i}>u_{p}$ ), the value of $\theta_{q-1}$ is zero. Hence we must simply evaluate

$$
\begin{aligned}
& (-1)^{q} \theta_{q-1}\left(J u_{p} \otimes u_{1}^{\otimes m_{1}} \otimes \cdots \otimes u_{p}^{\otimes m_{p}-1} \otimes x\right) \\
& \quad+\theta_{q-1}\left(J \otimes u_{1}^{\otimes m_{1}} \otimes \cdots \otimes u_{p}^{\otimes m_{p}-1} \otimes u_{p}(x)\right) .
\end{aligned}
$$

If $m_{p}>1$, then it is again clear that both of the terms above are zero. Hence, we must consider the case that $m_{p}=1$. Since $u_{p}<j_{s}, j_{s}$ is the $(s+1)$ th factor of $J u_{p}$, so if $u_{p-1} \geq j_{s}$, we have

$$
\begin{aligned}
& (-1)^{q} \theta_{q-1}\left(J u_{p} \otimes u_{1}^{\otimes m_{1}} \otimes \cdots \otimes u_{p-1}^{\otimes m_{p-1}} \otimes x\right)
\end{aligned}
$$

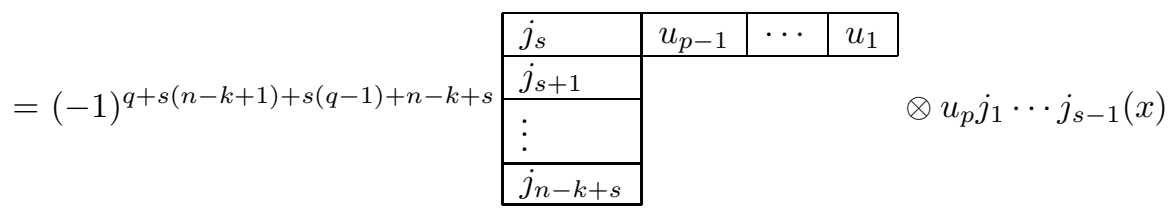

while

$$
\begin{aligned}
& \theta_{q-1}\left(J \otimes u_{1}^{\otimes m_{1}} \otimes \cdots \otimes u_{p}^{\otimes m_{p}-1} \otimes u_{p}(x)\right)
\end{aligned}
$$

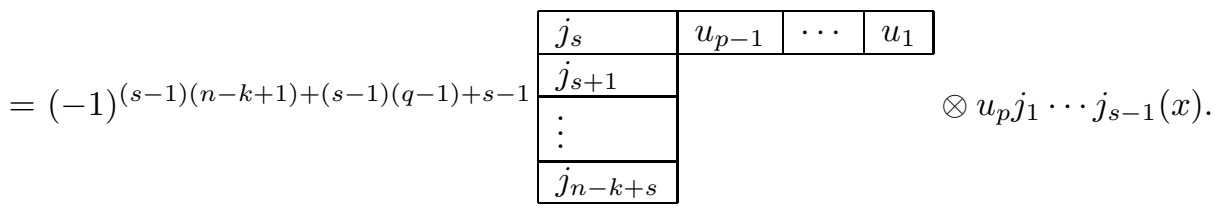

These have opposite sign, so the sum is zero. Of course, if $u_{p-1}<j_{s}$, each of the terms above is zero, and we have the result in the case that $u_{p}<j_{s}$. 
Now assume that $u_{p} \geq j_{s}$. We will assume the strict inequality; equality requires a separate, but easy, discussion. In this case, we have

$$
\begin{aligned}
& \partial_{T} \theta_{q}(Y)
\end{aligned}
$$

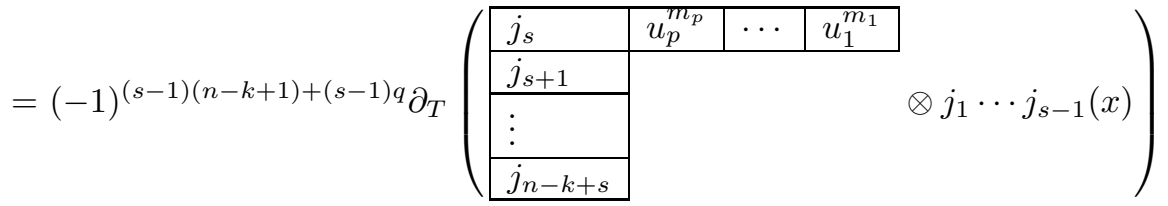

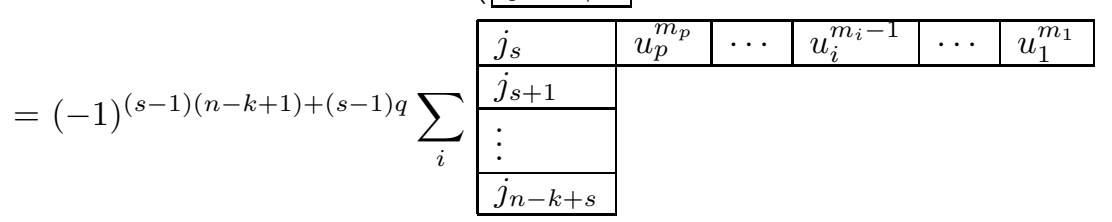

$$
\begin{aligned}
& \otimes u_{i} j_{1} \cdots j_{s-1}(x)
\end{aligned}
$$

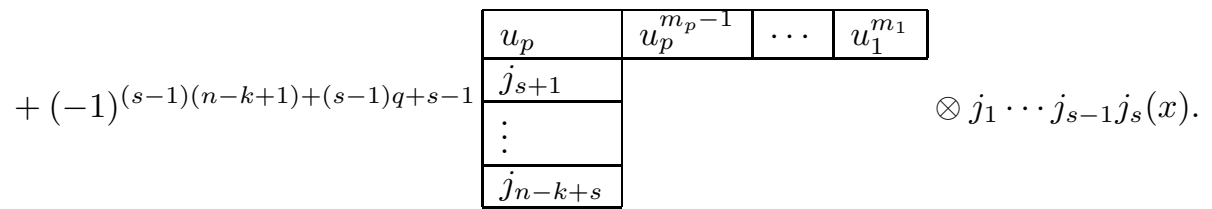

But we have

$$
\begin{aligned}
& \theta_{q-1}\left(J \otimes u_{1}^{\otimes m_{1}} \otimes \cdots \otimes u_{i}^{\otimes m_{i}-1} \otimes \cdots \otimes u_{p}^{\otimes m_{p}} \otimes u_{i}(x)\right)
\end{aligned}
$$

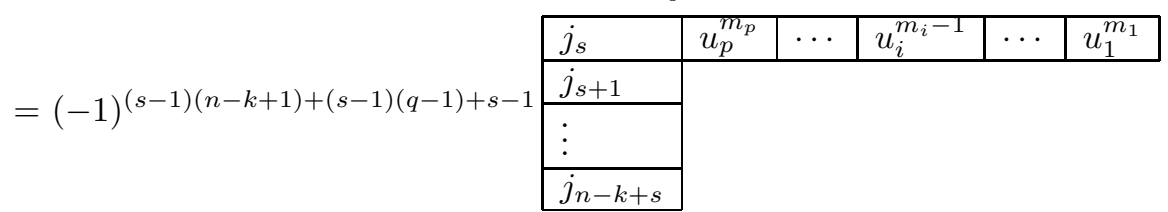

$$
\begin{aligned}
& \otimes u_{i} j_{1} \cdots j_{s-1}(x),
\end{aligned}
$$

which agrees (when we sum) with the first term for $\partial_{T} \theta_{q}(Y)$ and, if $u_{q} \geq j_{s+1}$ (in which case $u_{i} \geq j_{s+1}$ for all $\left.i\right)$,

$$
\begin{aligned}
& (-1)^{q} \sum_{i} \theta_{q-1}\left(J u_{i} \otimes u_{1}^{\otimes m_{1}} \otimes \cdots \otimes u_{i}^{\otimes m_{i}-1} \otimes \cdots \otimes u_{p}^{\otimes m_{p}} \otimes x\right)
\end{aligned}
$$

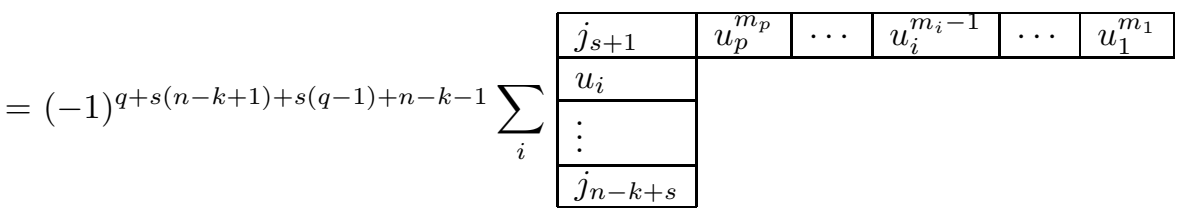

$$
\begin{aligned}
& \otimes j_{1} \cdots j_{s}(x) .
\end{aligned}
$$

However, if $u_{p} \geq j_{s+1}$, then the term

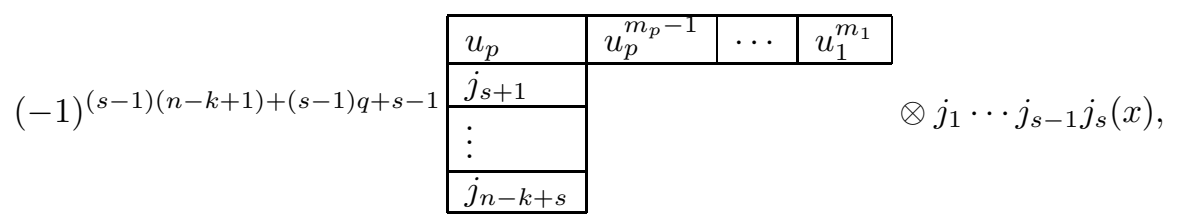

which arises in the calculation of $\partial_{T} \theta_{q}(Y)$, is not a standard tableau: it must be straightened. When straightened, it gives precisely the sum immediately above it. 
If, though, we assume that $j_{s}<u_{p}<j_{s+1}$, then

$$
\theta_{q-1}\left(J u_{i} \otimes u_{1}^{\otimes m_{1}} \otimes \cdots \otimes u_{i}^{\otimes m_{i}-1} \otimes \cdots \otimes u_{p}^{\otimes m_{p}} \otimes x\right)=0
$$

for all $i \neq p$, and in that case we must simply compute

$$
\theta_{q-1}\left(J u_{p} \otimes u_{1}^{\otimes m_{1}} \otimes \cdots \otimes u_{p-1}^{\otimes m_{p-1}} \otimes x\right) .
$$

This is easily seen to be equal to the term displayed immediately above, and the proof is complete.

Having obtained the map $\theta$, we are now in a position to transfer the homothety homotopy on the fat complex. That is, we have the homotopy $s$ that takes $\mathbf{C}(k ; f)$ to itself with the property that $\partial s+s \partial=\mu$, where $\mu$ is a given maximal minor of our original map. Let us denote the complex $\mathbf{C}(k ; f)$ by $Y$, and the slim complex $\mathbf{T}(k ; f)$ by $X$. Let us denote the boundary map on $X$ by $\partial_{X}$ and that on $Y$ by $\partial_{Y}$. Then we have the following lemma.

Lemma 3.4. Let $X$ and $Y$ be complexes, and let $\alpha: X \rightarrow Y$ and $\theta: Y \rightarrow X$ be maps of complexes such that $\theta \alpha=i d_{X}$. Let $s: Y \rightarrow Y$ be a homotopy on $Y$ which makes the scalar $\mu$ homotopic to zero on $Y$. Then the map $\tau=\theta$ s $\alpha: X \rightarrow X$ is a homotopy on $X$ carrying $\mu$ to zero.

Proof. First we have to show that $\partial_{X} \tau_{0}=\mu$. But $\partial_{X} \tau_{0}=\partial_{X} \theta_{1} s_{0} \alpha_{0}=\theta_{0} \partial_{Y} s_{0} \alpha_{0}=$ $\theta_{0} \mu \alpha_{0}=\mu \theta_{0} \alpha_{0}=\mu$. For $i>0$, we have to show that

$$
\partial_{X} \tau_{i}+\tau_{i-1} \partial_{X}=\mu
$$

But here again we have

$$
\begin{aligned}
\partial_{X} \tau_{i} & =\partial_{X} \theta_{i+1} s_{i} \alpha_{i}=\theta_{i} \partial_{Y} s_{i} \alpha_{i}=\theta_{i}\left(\mu-s_{i-1} \partial_{Y}\right) \alpha_{i} \\
& =\mu-\theta_{i} s_{i-1} \partial_{Y} \alpha_{i}
\end{aligned}
$$

while

$$
\tau_{i-1} \partial_{X}=\theta_{i} s_{i-1} \alpha_{i-1} \partial_{X}=\theta_{i} s_{i-1} \partial_{Y} \alpha_{i}
$$

and this does it.

As a result of this, we now know that our slim complexes carry the desired homotopy.

\section{The homotopy EQUivalence of the COMPLEXeS}

In this section we prove that the maps $\alpha$ and $\theta$ are inverses of each other up to homotopy. That is, we know that $\theta_{q} \alpha_{q}=i d$. What we want to show, then, is that there is a homotopy, $\eta$, on the fat complex, $\mathbf{C}(k ; f)$, such that

$$
\partial_{C} \eta+\eta \partial_{C}=1-\alpha \theta
$$

In order to define this homotopy, we have to introduce some auxiliary maps that are closely related to the maps $\alpha$ and $\theta$ defined in the previous section.

Definition of the maps $\theta_{q}^{\prime}$ : For each $q \geq 0$, we define the map

$$
\theta_{q}^{\prime}: \Lambda^{n-k+s_{0}} G^{*} \otimes \Lambda^{s_{1}} G^{*} \otimes \cdots \otimes \Lambda^{s_{q}} G^{*} \rightarrow K_{\left(q+1,1^{n-k}\right)} G^{*} \otimes \Lambda^{|s|-q-1} G^{*}
$$


as follows: if all the $s_{i}$ are equal to 1 for $i>0$, we adopt the notation of the previous section and set

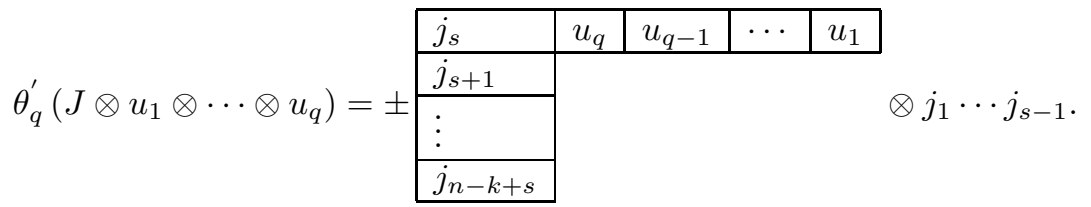

We still adhere to the convention that we adopted in the definition of the maps $\theta_{q}$ : the tableau is to be read as zero if the top row is not weakly increasing. For higher degree terms, we do as we did for the maps $\theta_{q}$ : if $U_{t+1}=v \wedge W$ is a basis element of $\Lambda^{s_{t+1}} G^{*}$, and $s_{t+2}=\cdots=s_{q}=1$, we set

$$
\begin{aligned}
& \theta_{q}^{\prime}\left(J \otimes U_{1} \otimes \cdots \otimes U_{t+1} \otimes \cdots \otimes U_{q}\right) \\
= & \theta_{q}^{\prime}\left(J \otimes U_{1} \otimes \cdots \otimes U_{t} v \otimes W \otimes \cdots \otimes U_{q}\right) \\
& \pm \theta_{q}^{\prime}\left(J \otimes U_{1} \otimes \cdots \otimes U_{t} \otimes v \otimes U_{t+2} \otimes \cdots \otimes U_{q}\right) W .
\end{aligned}
$$

The notation $\theta_{q}^{\prime}\left(J \otimes U_{1} \otimes \cdots \otimes U_{t} \otimes v \otimes U_{t+2} \otimes \cdots \otimes U_{q}\right) W$ is to be interpreted as follows: the element $\theta_{q}^{\prime}\left(J \otimes U_{1} \otimes \cdots \otimes U_{t} \otimes v \otimes U_{t+2} \otimes \cdots \otimes U_{q}\right)$ is in $K_{\left(q+1,1^{n-k}\right)} G^{*} \otimes \Lambda^{|s|-s_{t+1}-q} G^{*}$ while $W \in \Lambda^{s_{t+1}-1} G^{*}$. Our notation indicates that we are to multiply the element of $\Lambda^{|s|-s_{t+1}-q} G^{*}$ by $W$ and end up in $K_{\left(q+1,1^{n-k}\right)} G^{*} \otimes \Lambda^{|s|-q-1} G^{*}$ as we are supposed to. The signs signified by \pm are equal to those for the corresponding maps $\theta_{q}$.

Definition of the maps $\alpha_{q}^{\prime}$ : For each $q \geq 0$ we define the map

$$
\alpha_{q}^{\prime}: K_{\left(q+1,1^{n-k}\right)} G^{*} \rightarrow \Lambda^{n-k+1} G^{*} \otimes \Lambda^{1} G^{*} \otimes \cdots \otimes \Lambda^{1} G^{*}
$$

as the composition

$$
K_{\left(q+1,1^{n-k}\right)} G^{*} \hookrightarrow \Lambda^{n-k+1} G^{*} \otimes D_{q} G^{*} \rightarrow \Lambda^{n-k+1} G^{*} \otimes \underbrace{D_{1} G^{*} \otimes \cdots \otimes D_{1} G^{*}}_{q} .
$$

As the reader can see, this is just the map $\alpha_{q}$ with the exterior power of $F$ stripped away.

In addition to these maps, we introduce one more piece of notation. If $u_{1}, u_{2}, \ldots$,

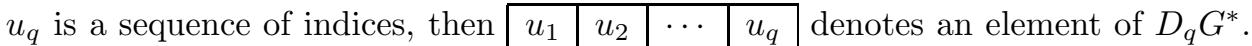
We denote by $\Delta\left(\begin{array}{l|l|l|l|}\hline u_{1} & u_{2} & \cdots & u_{q}\end{array}\right)$ the total diagonalization of this element in $\underbrace{D_{1} G^{*} \otimes \cdots \otimes D_{1} G^{*}}_{q}$. Keep in mind that, because of our earlier convention on reading tableaux, this is zero unless we have $u_{1} \leq u_{2} \leq \cdots \leq u_{q}$.

Now we are ready to define the homotopy $\eta: \mathbf{C}(k ; f) \rightarrow \mathbf{C}(k ; f)$.

Definition of the homotopy: For the sake of notational convenience, we start the homotopy with $\eta_{-2}: \Lambda^{k} G \rightarrow \Lambda^{k} F$, and set it equal to zero. Similarly, we set $\eta_{-1}: \Lambda^{k} F \rightarrow \sum_{s>1} \Lambda^{n-k+s} G^{*} \otimes \Lambda^{n+s} F$ to be zero. We define $\eta_{0}: \sum_{s \geq 1} \Lambda^{n-k+s} G^{*} \otimes \Lambda^{n+s} F \rightarrow \sum_{s_{i} \geq 1} \Lambda^{n-k+s_{0}} G^{*} \otimes \Lambda^{s_{1}} G^{*} \otimes \Lambda^{n+|s|} F$ as

$$
\eta_{0}(J \otimes x)=\theta_{0}^{\prime}(J) \otimes x .
$$


For an element $A=J \otimes u_{1} \otimes \cdots \otimes u_{q} \otimes x$, with all the $u_{i}$ of degree one, and $J$ of degree $n-k+s$, we define

$$
\begin{aligned}
\eta_{q}(A)= & \sum_{i=0}^{q}(-1)^{i}(\alpha_{i}^{\prime} \otimes \underbrace{1 \otimes \cdots \otimes 1}_{q-i+2})(\theta_{i}^{\prime} \otimes \underbrace{1 \otimes \cdots \otimes 1}_{q-i+1}) \\
& \left(J \otimes \Delta\left(\begin{array}{|l|l|l|l|}
u_{q} & u_{q-1} & \cdots & u_{1} \\
\hline
\end{array}\right) x\right) .
\end{aligned}
$$

Assume that $\eta_{q}$ has been defined on elements $Y=J \otimes U_{1} \otimes \cdots \otimes U_{q} \otimes x$ with $U_{1}, \ldots, U_{t}$ of arbitrary degree, $U_{t+1}$ of degree $\leq r$, and $U_{i}$ of degree one for $i>t+1$. Let $A$ be an element: $A=J \otimes U_{1} \otimes \cdots \otimes U_{t} \otimes U_{t+1} \otimes u_{t+2} \otimes$ $\cdots \otimes u_{q} \otimes x$ with $U_{1}, \ldots, U_{t}$ basis elements of arbitrary degree, $U_{t+1}=v W$ with degree $(v)=1$, degree $(W)=r$, and the degree of $u_{i}$ equal to one for $i>t+1$. We define

$$
\begin{aligned}
& \eta_{q}(A)
\end{aligned}
$$

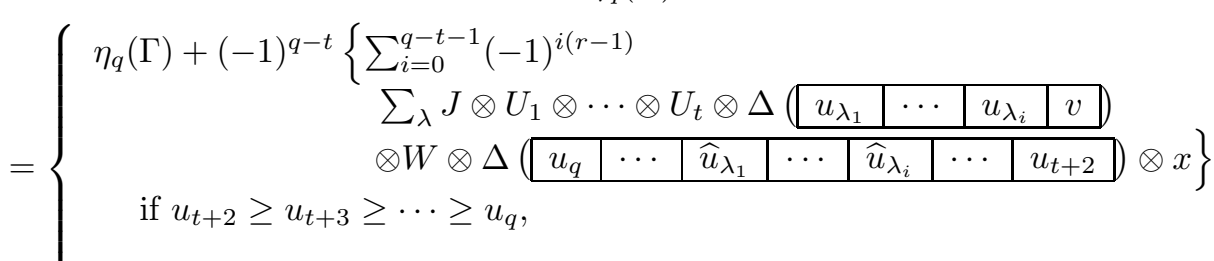

where

$$
\begin{aligned}
\Gamma= & J \otimes U_{1} \otimes \cdots \otimes U_{t} v \otimes W \otimes u_{t+2} \otimes \cdots \otimes u_{q} \otimes x \\
& +(-1)^{r(q-t-1)} J \otimes U_{1} \otimes \cdots \otimes U_{t} \otimes v \otimes u_{t+2} \otimes \cdots \otimes u_{q} \otimes W(x),
\end{aligned}
$$

and $\lambda=\left(\lambda_{1}, \ldots, \lambda_{i}\right)$ is a strictly descending sequence of indices between $t+2$ and $q$.

As in the definition of the maps $\theta_{q}$, we resort to this inductive definition, making heavy use of the fact that there is no upper bound on the degree of $J$ at any point.

The fact that this does provide the desired homotopy is not immediately obvious. In fact, the rest of the section is devoted to the proof of this fact. First we will prove two lemmas which are special cases of the main theorem.

Lemma 4.1. Let $Y=J \otimes U_{1} \otimes \cdots \otimes U_{t} \otimes u_{t+1} \otimes \cdots \otimes u_{q} \otimes x$, with $u_{i}$ of degree one for $i>t$. Then $\eta_{q-1} \partial_{C}(Y)=Y$ unless $u_{q} \leq u_{q-1} \leq \cdots \leq u_{t+1}$.

Proof. First, we must prove this result for $q=2$. In that case we have, if $Y=$ $J \otimes u_{1} \otimes u_{2} \otimes x$,

$$
\begin{aligned}
\eta_{1} \partial_{C}(Y)= & \eta_{1}\left(J u_{1} \otimes u_{2} \otimes x\right) \\
& -\eta_{1}\left(J \otimes u_{1} u_{2} \otimes x\right)+\eta_{1}\left(J \otimes u_{1} \otimes u_{2}(x)\right) .
\end{aligned}
$$

But if we assume that $u_{2}>u_{1}$, then

$$
\eta_{1}\left(J \otimes u_{1} u_{2} \otimes x\right)=\eta_{1}\left(J u_{1} \otimes u_{2} \otimes x\right)+\eta_{1}\left(J \otimes u_{1} \otimes u_{2}(x)\right)-J \otimes u_{1} \otimes u_{2} \otimes x,
$$

and so for $q=2$, we are done. Now we proceed to prove this result by induction on $q$. 
Suppose our first 'wrong' inequality occurs in the last place, that is, suppose that $u_{q-1}<u_{q}$. We immediately see that we have the result for

$$
\begin{aligned}
& \eta_{q-1} \partial_{C}(Y) \\
= & \sum_{i=1}^{q-2}(-1)^{q-i+1} \eta_{q-1}\left(J \otimes U_{1} \otimes \cdots \otimes U_{i-1} U_{i} \otimes \cdots \otimes u_{q-1} \otimes u_{q} \otimes x\right) \\
& +\eta_{q-1}\left(J \otimes U_{1} \otimes \cdots \otimes U_{q-2} u_{q-1} \otimes u_{q} \otimes x\right) \\
& \quad-\eta_{q-1}\left(J \otimes U_{1} \otimes \cdots \otimes U_{q-2} \otimes u_{q-1} u_{q} \otimes x\right) \\
& +\eta_{q-1}\left(J \otimes U_{1} \otimes \cdots \otimes u_{q-1} \otimes u_{q}(x)\right) .
\end{aligned}
$$

By our definition of $\eta_{q-1}$, we see that the terms

$$
\eta_{q-1}\left(J \otimes U_{1} \otimes \cdots \otimes U_{i-1} U_{i} \otimes \cdots \otimes u_{q-1} \otimes u_{q} \otimes x\right)
$$

are zero. On the other hand, we know that

$$
\begin{array}{ll} 
& -\eta_{q-1}\left(J \otimes U_{1} \otimes \cdots \otimes U_{q-2} \otimes u_{q-1} u_{q} \otimes x\right) \\
= & -\eta_{q-1}\left(J \otimes U_{1} \otimes \cdots \otimes U_{q-2} u_{q-1} \otimes u_{q} \otimes x\right) \\
& -\eta_{q-1}\left(J \otimes U_{1} \otimes \cdots \otimes U_{q-2} \otimes u_{q-1} \otimes u_{q}(x)\right) \\
& +J \otimes U_{1} \otimes \cdots \otimes u_{q-1} \otimes u_{q} \otimes x .
\end{array}
$$

Thus the surviving terms above are simply $Y$, and we are done.

Now suppose that for some $l$ with $t+1<l<q$, we have $u_{l-1}<u_{l}$, but $u_{q} \leq u_{q-1} \leq \cdots \leq u_{l}$. As in the previous section, we will rewrite $Y$ as follows:

$$
Y=J \otimes U_{1} \otimes \cdots \otimes U_{l-2} \otimes u \otimes u_{1}^{\otimes m_{1}} \otimes \cdots \otimes u_{p}^{\otimes m_{p}} \otimes x
$$

with $m_{1}+\cdots+m_{p}=q-l+1, u<u_{1}$, and $u_{p}<\cdots<u_{1}$. Set $\mu_{i}=m_{p}+\cdots+m_{i}$; we have

$$
\begin{aligned}
& \quad \eta_{q-1} \partial_{C}(Y) \\
& =\sum_{i=1}^{l-2}(-1)^{q-i+1} \eta_{q-1}\left(J \otimes U_{1} \otimes \cdots \otimes U_{i-1} U_{i} \otimes \cdots \otimes u \otimes u_{1}^{\otimes m_{1}} \otimes \cdots \otimes u_{p}^{\otimes m_{p}} \otimes x\right) \\
& \quad+(-1)^{q-l+2} \eta_{q-1}\left(J \otimes U_{1} \otimes \cdots \otimes U_{l-2} u \otimes u_{1}^{\otimes m_{1}} \otimes \cdots \otimes u_{p}^{\otimes m_{p}} \otimes x\right) \\
& \quad+(-1)^{q-l+1} \eta_{q-1}\left(J \otimes U_{1} \otimes \cdots \otimes U_{l-2} \otimes u u_{1} \otimes u_{1}^{\otimes m_{1}-1} \otimes \cdots \otimes u_{p}^{\otimes m_{p}} \otimes x\right) \\
& \quad+\sum_{i=2}^{p}(-1)^{\mu_{i}} \eta_{q-1}\left(J \otimes U_{1} \otimes \cdots \otimes u \otimes u_{1}^{\otimes m_{1}} \otimes \cdots \otimes u_{i-1}^{\otimes m_{i-1}-1}\right. \\
& \left.\quad \otimes u_{i-1} u_{i} \otimes u_{i}^{\otimes m_{i}-1} \otimes \cdots \otimes u_{p}^{\otimes m_{p}} \otimes x\right) \\
& \quad+\eta_{q-1}\left(J \otimes U_{1} \otimes \cdots \otimes U_{l-2} \otimes u \otimes u_{1}^{\otimes m_{1}} \otimes \cdots \otimes u_{p-1}^{\otimes m_{p-1}} \otimes u_{p}^{\otimes m_{p}-1} \otimes u_{p}(x)\right) .
\end{aligned}
$$

By the same argument as above, we see that the summands from 1 to $l-2$ are all zero. Also, the last term is zero, since we are assuming now that $l<q$ (i.e., that 
$u_{1}>u_{p}$, or, if $p=1$, then $\left.m_{p}>1\right)$. So this leaves us with the problem of evaluating

$$
\begin{gathered}
(-1)^{q-l+2} \eta_{q-1}\left(J \otimes U_{1} \otimes \cdots \otimes U_{l-2} u \otimes u_{1}^{\otimes m_{1}} \otimes \cdots \otimes u_{p}^{\otimes m_{p}} \otimes x\right) \\
+(-1)^{q-l+1} \eta_{q-1}\left(J \otimes U_{1} \otimes \cdots \otimes U_{l-2} \otimes u u_{1} \otimes u_{1}^{\otimes m_{1}-1} \otimes \cdots \otimes u_{p}^{\otimes m_{p}} \otimes x\right) \\
+\sum_{i=2}^{p}(-1)^{\mu_{i}} \eta_{q-1}\left(J \otimes U_{1} \otimes \cdots \otimes u \otimes u_{1}^{\otimes m_{1}} \otimes \cdots \otimes u_{i-1}^{\otimes m_{i-1}-1}\right. \\
\left.\otimes u_{i-1} u_{i} \otimes u_{i}^{\otimes m_{i}-1} \otimes \cdots \otimes u_{p}^{\otimes m_{p}} \otimes x\right) .
\end{gathered}
$$

Now

$$
\begin{aligned}
& (-1)^{q-l+1} \eta_{q-1}\left(J \otimes U_{1} \otimes \cdots \otimes U_{l-2} \otimes u u_{1} \otimes u_{1}^{\otimes m_{1}-1} \otimes \cdots \otimes u_{p}^{\otimes m_{p}} \otimes x\right) \\
& =(-1)^{q-l+1} \eta_{q-1}\left(J \otimes U_{1} \otimes \cdots \otimes U_{l-2} u \otimes u_{1} \otimes u_{1}^{\otimes m_{1}-1} \otimes \cdots \otimes u_{p}^{\otimes m_{p}} \otimes x\right) \\
& +(-1)^{q-l+1+q-l} \eta_{q-1}\left(J \otimes U_{1} \otimes \cdots \otimes U_{l-2} \otimes u \otimes u_{1}^{\otimes m_{1}-1} \otimes \cdots \otimes u_{p}^{\otimes m_{p}} \otimes u_{1}(x)\right) \\
& +\sum_{\rho \geq 0} \sum_{\left(n_{p}, \ldots, n_{2}\right)} J \otimes U_{1} \otimes \cdots \otimes U_{l-2} \otimes \Delta\left(\begin{array}{|l|l|l|l}
\hline u_{p}^{n_{p}} & \cdots & u_{2}^{n_{2}} & u \\
\hline
\end{array}\right) \otimes u_{1} \\
& \otimes \Delta\left(\begin{array}{|l|l|l|l}
\hline u_{p}^{m_{p}-n_{p}} & \cdots & u_{2}^{m_{2}-n_{2}} & u_{1}^{m_{1}-1} \\
\hline
\end{array}\right) \otimes x,
\end{aligned}
$$

where $\rho=n_{2}+\cdots+n_{p}$ and $\left(n_{p}, \ldots, n_{2}\right)$ runs over all sequences of non-negative integers such that $n_{i} \leq m_{i}$ for $i=2, \ldots, p$ 每 Consequently, we see that

$$
\begin{aligned}
& \eta_{q-1} \partial_{C}(Y) \\
&= \sum_{i=2}^{p}(-1)^{\mu_{i}} \eta_{q-1}\left(J \otimes U_{1} \otimes \cdots \otimes u \otimes u_{1}^{\otimes m_{1}} \otimes \cdots \otimes u_{i-1}^{\otimes m_{i-1}-1}\right. \\
&\left.\qquad u_{i-1} u_{i} \otimes u_{i}^{\otimes m_{i}-1} \otimes \cdots \otimes u_{p}^{\otimes m_{p}} \otimes x\right) \\
&-\eta_{q-1}\left(J \otimes U_{1} \otimes \cdots \otimes U_{l-2} \otimes u \otimes u_{1}^{\otimes m_{1}-1} \otimes \cdots \otimes u_{p}^{\otimes m_{p}} \otimes u_{1}(x)\right) \\
&+\sum_{\rho \geq 0} \sum_{\left(n_{p}, \ldots, n_{2}\right)} J \otimes U_{1} \otimes \cdots \otimes U_{l-2} \otimes \Delta\left(\begin{array}{|l|l|l|l|}
\hline u_{p}^{n_{p}} & \cdots & u_{2}^{n_{2}} & u
\end{array}\right) \otimes u_{1} \\
& \otimes \Delta\left(\begin{array}{|l|l|l|l|}
\hline u_{p}^{m_{p}-n_{p}} & \cdots & u_{2}^{m_{2}-n_{2}} & u_{1}^{m_{1}-1} \\
\hline
\end{array}\right.
\end{aligned}
$$

(Note that since all of this action is taking place above $t$-level, we are never encountering anything but linear terms as we proceed. Notice too that in our last double sum, we have not put in any sign that depends upon $\rho$; that is, because we are assuming our formula for our reductions has a sign $\rho(r-1)$ in it, and so these signs disappear when $r=1$.)

Clearly what remains to be done is to calculate

$$
\begin{array}{r}
\sum_{i=2}^{p}(-1)^{\mu_{i}} \eta_{q-1}\left(J \otimes U_{1} \otimes \cdots \otimes u \otimes u_{1}^{\otimes m_{1}} \otimes \cdots \otimes u_{i-1}^{\otimes m_{i-1}-1} \otimes u_{i-1} u_{i} \otimes u_{i}^{\otimes m_{i}-1}\right. \\
\left.\otimes \cdots \otimes u_{p}^{\otimes m_{p}} \otimes x\right) .
\end{array}
$$

\footnotetext{
${ }^{3}$ We should explain that we use the notation \begin{tabular}{|l|l|l|l|}
\hline$u_{p}^{n_{p}}$ & $\cdots$ & $u_{2}^{n_{2}}$ & $u$ \\
\hline
\end{tabular} to indicate the element of the divided power algebra represented by this tableau. Thus the exponents mean that the element is repeated in the tableau. We could also write $u_{p}^{\left(n_{p}\right)} \cdots u_{2}^{\left(n_{2}\right)} u$ but, while this is equal to $u u_{p}^{\left(n_{p}\right)} \cdots u_{2}^{\left(n_{2}\right)}$ in the divided power algebra, the tableau \begin{tabular}{|l|l|l|l|}
\hline$u$ & $u_{p}^{n_{p}}$ & $\cdots$ & $u_{2}^{n_{2}}$ \\
\hline
\end{tabular} element 0 .
} 
As in the proof of Theorem 3.3, we are going to use 'reduction formulas' to move our element $u_{i}$ to the left until it hits up against $u_{1}$. As we do this, certain terms are going to be sent to zero under $\eta_{q-1}$. As opposed to the situation in Theorem 3.3 , however, we will pick up terms involving total diagonalizations; these are the 'correction terms' that figure into the definition of our homotopy. To illustrate, we see that

$$
\begin{aligned}
& \eta_{q-1}\left(J \otimes U_{1} \otimes \cdots \otimes u \otimes u_{1}^{\otimes m_{1}} \otimes \cdots \otimes u_{i-1}^{\otimes m_{i-1}-1} \otimes u_{i-1} u_{i}\right. \\
& \left.\otimes u_{i}^{\otimes m_{i}-1} \otimes \cdots \otimes u_{p}^{\otimes m_{p}} \otimes x\right) \\
& =-\eta_{q-1}\left(J \otimes U_{1} \otimes \cdots \otimes u \otimes u_{1}^{\otimes m_{1}} \otimes \cdots \otimes u_{i-1}^{\otimes m_{i-1}-1} \otimes u_{i} u_{i-1}\right. \\
& \left.\otimes u_{i}^{\otimes m_{i}-1} \otimes \cdots \otimes u_{p}^{\otimes m_{p}} \otimes x\right) \\
& =-\eta_{q-1} J \otimes U_{1} \otimes \cdots \otimes u \otimes u_{1}^{\otimes m_{1}} \otimes \cdots \otimes u_{i-1}^{\otimes m_{i-1}-2} \otimes u_{i-1} u_{i} \otimes u_{i-1} \\
& \left.\otimes u_{i}^{\otimes m_{i}-1} \otimes \cdots \otimes u_{p}^{\otimes m_{p}} \otimes x\right) \\
& +(-1)^{\mu_{i}} \eta_{q-1}\left(J \otimes U_{1} \otimes \cdots \otimes u \otimes u_{1}^{\otimes m_{1}} \otimes \cdots \otimes u_{i-1}^{\otimes m_{i-1}-1} \otimes u_{i}^{\otimes m_{i}}\right. \\
& \left.\otimes \cdots \otimes u_{p}^{\otimes m_{p}} \otimes u_{i-1}(x)\right) \\
& -(-1)^{\mu_{i}} \sum_{\rho_{i} \geq 0} \sum_{\left(n_{p}, \ldots, n_{i}\right)} J \otimes U_{1} \otimes \cdots \otimes u \otimes u_{1}^{\otimes m_{1}} \otimes \cdots \otimes u_{i-1}^{\otimes m_{i-1}-1} \\
& \otimes \Delta\left(\begin{array}{|l|l|l|l|}
\hline u_{p}^{n_{p}} & \cdots & u_{i}^{n_{i}} & u_{i} \\
\hline
\end{array}\right) \otimes u_{i-1} \\
& \otimes \Delta\left(\begin{array}{|l|l|l}
\hline u_{p}^{m_{p}-n_{p}} & \cdots & u_{i}^{m_{i}-n_{i}-1} \\
\hline
\end{array}\right) \otimes x .
\end{aligned}
$$

We immediately see that the terms

$$
\begin{aligned}
&(-1)^{\mu_{i}} \eta_{q-1}\left(J \otimes U_{1} \otimes \cdots \otimes u \otimes u_{1}^{\otimes m_{1}} \otimes \cdots \otimes u_{i-1}^{\otimes m_{i-1}-1} \otimes u_{i}^{\otimes m_{i}}\right. \\
&\left.\otimes \cdots \otimes u_{p}^{\otimes m_{p}} \otimes u_{i-1}(x)\right)
\end{aligned}
$$

are zero for $i>2$, and that for $i=2$, the term is zero unless $m_{1}=1$. Since this term is to be multiplied by $(-1)^{\mu_{i}}$, it occurs with a positive sign, and cancels the corresponding term in the original calculation of $\eta_{q-1} \partial_{C}(Y)$ above.

We now want to continue to eliminate the quadratic term (i.e., the one involving $\left.\otimes u_{i-1} u_{i} \otimes u_{i-1} \otimes\right)$; the other remaining term, although by no means elegant, is here to stay. But as in Theorem 3.3, when we apply our reduction process, we will get another quadratic term, then a term which is carried to zero by $\eta_{q-1}$ (because we will have $u_{i}$ to the left of $u_{i-1}$ ), and another correction term (which cannot be discarded). Continuing with this type of reduction, and letting $Z$ denote $J \otimes U_{1} \otimes \cdots \otimes U_{l-2}$, we finally arrive at the conclusion that

$$
\begin{aligned}
& (-1)^{\mu_{i}} \eta_{q-1}\left(Z \otimes u \otimes u_{1}^{\otimes m_{1}} \otimes \cdots \otimes u_{i-1}^{\otimes m_{i-1}-1} \otimes u_{i-1} u_{i} \otimes u_{i}^{\otimes m_{i}-1}\right. \\
& \left.\otimes \cdots \otimes u_{p}^{\otimes m_{p}} \otimes x\right) \\
& =(-1)^{q-l+1} \eta_{q-1}\left(Z \otimes u u_{i} \otimes u_{1}^{\otimes m_{1}} \otimes \cdots \otimes u_{i-1}^{\otimes m_{i-1}} \otimes u_{i}^{\otimes m_{i}-1} \otimes \cdots \otimes u_{p}^{\otimes m_{p}} \otimes x\right) \\
& -\sum_{\lambda=1}^{i-1} \sum_{\alpha_{\lambda}=1}^{m_{\lambda}} Z \otimes u \otimes u_{1}^{\otimes m_{1}} \otimes \cdots \otimes u_{\lambda-1}^{\otimes m_{\lambda-1}} \\
& \otimes u_{\lambda}^{\otimes m_{\lambda}-\alpha_{\lambda}} \otimes \Delta\left(\begin{array}{|l|l|l|l|}
\hline u_{p}^{n_{p}} & \cdots & u_{i}^{n_{i}} & u_{i} \\
\hline
\end{array}\right) \otimes u_{\lambda} \\
& \otimes \Delta\left(\begin{array}{|l|l|l|l|l|l|}
\hline u_{p}^{m_{p}-n_{p}} & \cdots & u_{i}^{m_{i}-n_{i}-1} & u_{i-1}^{m_{i-1}} & \cdots & u_{\lambda}^{\alpha_{\lambda}-1} \\
\hline
\end{array}\right) \otimes x,
\end{aligned}
$$


so that we see that (making a few changes in our indices of summation in the "non-eta" terms)

$$
\begin{aligned}
& \eta_{q-1} \partial(Y) \\
& =\sum Z \otimes \Delta\left(\begin{array}{|l|l|l|l|}
\hline u_{p}^{n_{p}} & \cdots & u_{2}^{n_{2}} & u \\
\hline
\end{array}\right. \\
& \otimes \Delta\left(\begin{array}{|l|l|l|l}
\hline u_{p}^{m_{p}-n_{p}} & \cdots & u_{2}^{m_{2}-n_{2}} & u_{1}^{m_{1}-1} \\
\hline
\end{array}\right) \otimes x \\
& -\sum_{\lambda=i+1}^{p} \sum_{i=1}^{p-1} \sum_{\alpha_{i}=0}^{m_{i}-1} Z \otimes u \otimes u_{1}^{\otimes m_{1}} \otimes \cdots \otimes u_{i-1}^{\otimes m_{i-1}} \\
& \otimes u_{i}^{\otimes \alpha_{i}} \otimes \Delta\left(\begin{array}{|l|l|l}
\hline u_{p}^{\beta_{p}} & \cdots & u_{\lambda}^{\beta_{\lambda}+1} \\
\hline
\end{array}\right. \\
& \otimes u_{i} \otimes \Delta\left(\begin{array}{l|l|l|l|l|}
\hline u_{p}^{m_{p}-\beta_{p}} & \cdots & u_{\lambda}^{m_{\lambda}-\beta_{\lambda}-1} & \cdots & u_{i}^{m_{i}-\alpha_{i}-1} \\
\hline
\end{array}\right) \otimes x \\
& +(-1)^{q-l+1} \sum_{i=2}^{p} \eta_{q-1}\left(Z \otimes u u_{i} \otimes u_{1}^{\otimes m_{1}} \otimes \cdots \otimes u_{i-1}^{\otimes m_{i-1}} \otimes u_{i}^{\otimes m_{i}-1}\right. \\
& \left.\otimes \cdots \otimes u_{p}^{\otimes m_{p}} \otimes x\right),
\end{aligned}
$$

where $\beta_{\gamma} \geq 0$. Thus, what we have left is to evaluate

$$
(-1)^{q-l+1} \sum_{i=2}^{p} \eta_{q-1}\left(Z \otimes u u_{i} \otimes u_{1}^{\otimes m_{1}} \otimes \cdots \otimes u_{i-1}^{\otimes m_{i-1}} \otimes u_{i}^{\otimes m_{i}-1} \otimes \cdots \otimes u_{p}^{\otimes m_{p}} \otimes x\right),
$$

and this clearly depends on the relative sizes of $u$ and $u_{i}$ for $i=2, \ldots, p$.

First, let us assume that $u<u_{p}<\cdots<u_{1}$. In that case, we see that

$$
\begin{aligned}
& (-1)^{q-l+1} \sum_{i=2}^{p} \eta_{q-1}\left(Z \otimes u u_{i} \otimes u_{1}^{\otimes m_{1}} \otimes \cdots \otimes u_{i-1}^{\otimes m_{i-1}} \otimes u_{i}^{\otimes m_{i}-1} \otimes \cdots \otimes u_{p}^{\otimes m_{p}} \otimes x\right) \\
= & \sum_{i=2}^{p} Z \otimes u \otimes u_{i} \otimes \Delta\left(\begin{array}{|l|l|l|l|l}
\hline u_{p}^{m_{p}} & \cdots & u_{i}^{m_{i}-1} & \cdots & u_{1}^{m_{1}} \\
\hline
\end{array}\right) \otimes x .
\end{aligned}
$$

(The terms involving $\eta$ disappear because either $u$ or $u_{i}$ would appear before the $u_{1}$, and that would make $\eta$ vanish.) This then yields

$$
\begin{aligned}
& \eta_{q-1} \partial(Y)=Z \otimes u \otimes \sum_{i=1}^{p} u_{i} \otimes \Delta\left(\begin{array}{|l|l|l|l|l|}
\hline u_{p}^{m_{p}} & \cdots & u_{i}^{m_{i}-1} & \cdots & u_{1}^{m_{1}} \\
\hline
\end{array}\right) \otimes x \\
& -\sum_{\lambda=i+1}^{p} \sum_{i=1}^{p-1} \sum_{\alpha_{i}=0}^{m_{i}-1} Z \otimes u \otimes u_{1}^{\otimes m_{1}} \otimes \cdots \otimes u_{i-1}^{\otimes m_{i-1}} \\
& \otimes u_{i}^{\otimes \alpha_{i}} \otimes \Delta\left(\begin{array}{|l|l|l}
\hline u_{p}^{\beta_{p}} & \cdots & u_{\lambda}^{\beta_{\lambda}+1} \\
\hline
\end{array}\right. \\
& \otimes u_{i} \otimes \Delta\left(\begin{array}{|l|l|l|l|l|}
\hline u_{p}^{m_{p}-\beta_{p}} & \cdots & u_{\lambda}^{m_{\lambda}-\beta_{\lambda}-1} & \cdots & u_{i}^{m_{i}-\alpha_{i}-1} \\
\hline
\end{array}\right) \otimes x .
\end{aligned}
$$

A simple argument shows us that the term that we are subtracting is equal to all of the positive term except for the element $Y$. For we have

$$
\sum_{i=1}^{p} u_{i} \otimes \Delta\left(\begin{array}{|l|l|l|l|l}
\hline u_{p}^{m_{p}} & \cdots & u_{i}^{m_{i}-1} & \cdots & u_{1}^{m_{1}} \\
\hline
\end{array}\right)=\Delta\left(\begin{array}{|l|l|l|l|l|}
u_{p}^{m_{p}} & \cdots & u_{i}^{m_{i}} & \cdots & u_{1}^{m_{1}} \\
\hline
\end{array}\right),
$$


and this latter term runs through all $(q-l)$-fold tensor products of content $\left(m_{1}, \ldots\right.$, $m_{p}$ ). The terms that we are subtracting can clearly be seen to run through all such tensor products of the same content, except for the term $u_{1}^{\otimes m_{1}} \otimes \cdots \otimes u_{p}^{\otimes m_{p}}$. Thus, the difference is just $Y$. Let us record this fact as a separate identity:

$$
\begin{gathered}
Y=Z \otimes u \otimes \sum_{i=1}^{p} u_{i} \otimes \Delta\left(\begin{array}{|l|l|l|l|l|l}
\hline u_{p}^{m_{p}} & \cdots & u_{i}^{m_{i}-1} & \cdots & u_{1}^{m_{1}}
\end{array}\right) \otimes x \\
-\sum_{\lambda=i+1}^{p} \sum_{i=1}^{p-1} \sum_{\alpha_{i}=0}^{m_{i}-1} Z \otimes u \otimes u_{1}^{\otimes m_{1}} \otimes \cdots \otimes \\
u_{i-1}^{\otimes m_{i-1}} \otimes u_{i}^{\otimes \alpha_{i}} \otimes \Delta\left(\begin{array}{|l|l|l|l|l|}
\hline u_{p}^{\beta_{p}} & \cdots & u_{\lambda}^{\beta_{\lambda}+1}
\end{array}\right) \\
\otimes u_{i} \otimes \Delta\left(\begin{array}{ll|l|l|l}
\hline u_{p}^{m_{p}-\beta_{p}} & \cdots & u_{\lambda}^{m_{\lambda}-\beta_{\lambda}-1} & \cdots & u_{i}^{m_{i}-\alpha_{i}-1} \\
\hline
\end{array}\right.
\end{gathered}
$$

We will now give a full argument to handle the case that $u_{p}<u<u_{p-1}<$ $\cdots<u_{1}$. Once we do this, the general argument will be clear. The thing we must do in this situation is to see what gets added and what gets subtracted from the terms in (4.1) above. A term that gets added to the positive terms, due to the fact that we may now consider the tableau \begin{tabular}{|l|l|}
\hline$u_{p}^{\beta_{p}+1}$ & $u$
\end{tabular} , is $Z \otimes \Delta\left(\begin{array}{|l|l|l|}u_{p}^{\beta_{p}+1} & u \\
\hline\end{array} \otimes\right.$ $u_{1} \otimes \Delta\left(\begin{array}{|l|l|l|}\hline u_{p}^{m_{p}-\beta_{p}-1} & \cdots & u_{1}^{m_{1}} \\ \hline\end{array}\right) \otimes x$. Since we no longer have $Z \otimes u \otimes u_{p} \otimes$ $\Delta\left(\begin{array}{|l|l|l|l|l|}\hline u_{p}^{m_{p}-1} & \cdots & u_{i}^{m_{i}} & \cdots & u_{1}^{m_{1}} \\ \hline\end{array}\right) \otimes x$, we must subtract it from our positive terms. At the same time, we must consider the contribution from

$$
(-1)^{q-l+1} \eta_{q-1}\left(Z \otimes u u_{p} \otimes u_{1} \otimes \cdots \otimes u_{p-1} \otimes x\right),
$$

which is $-Z \otimes u_{p}^{\otimes \beta_{p}+1} \otimes u \otimes \Delta\left(\begin{array}{|l|l|l|}\hline u_{p}^{m_{p}-\beta_{p}-1} & \cdots & u_{1}^{m_{1}} \\ \hline\end{array}\right) \otimes x$. However, each of the terms

$$
(-1)^{q-l+1} \eta_{q-1}\left(Z \otimes u u_{i} \otimes u_{1} \otimes \cdots \otimes u_{i-1} \otimes u_{i+1} \otimes \cdots \otimes u_{p} \otimes x\right),
$$

with $1<i<p$, contributes a positive term that it did not contribute in the preceding case, namely

$$
Z \otimes \Delta\left(\begin{array}{|l|l|}
\hline u_{p}^{\beta_{p}+1} & u \\
\hline
\end{array}\right) \otimes u_{i} \otimes \Delta\left(\begin{array}{|l|l|l|l|l}
\hline u_{p}^{m_{p}-\beta_{p}-1} & \cdots & u_{i}^{m_{i}} & \cdots & u_{1}^{m_{1}} \\
\hline
\end{array}\right) \otimes x .
$$

But now it is easy to see that the terms added cancel out the terms subtracted, and this completes the proof in this case. Clearly, to attack the general case, we assume that $u_{p}<\cdots<u_{i+1} \leq u<u_{i}<\cdots<u_{1}$ and notice that in each step the terms added cancel those subtracted, as in the case just treated. This completes the proof of our lemma.

This next lemma tells us that our explicit definition of $\eta$ for terms all of whose elements are of degree one satisfies the homotopy identity.

Lemma 4.2. Let $Y=J \otimes u_{1} \otimes \cdots \otimes u_{q} \otimes x$, with all the $u_{i}$ of degree 1 . Then

$$
\partial_{C} \eta_{q}(Y)+\eta_{q-1} \partial_{C}(Y)=Y-\alpha_{q} \theta_{q}(Y) \text {. }
$$


Proof. We will again write $Y=J \otimes u_{1}^{\otimes m_{1}} \otimes \cdots \otimes u_{p}^{\otimes m_{p}} \otimes x$ with $u_{1}>\cdots>u_{p}$ and $\sum m_{i}=q$. By a process identical to the one used to prove the lemma above, and using the identity (4.1) above, it can be easily shown that

$$
\begin{aligned}
& \eta_{q-1} \partial_{C}(Y) \\
= & (-1)^{q} \sum_{i=1}^{p} \eta_{q-1}\left(J u_{i} \otimes u_{1}^{\otimes m_{1}} \otimes \cdots \otimes u_{i-1}^{\otimes m_{i-1}} \otimes u_{i}^{\otimes m_{i}-1} \otimes \cdots \otimes u_{p}^{\otimes m_{p}} \otimes x\right) \\
& +\sum_{i=1}^{p} \eta_{q-1}\left(J \otimes u_{1}^{\otimes m_{1}} \otimes \cdots \otimes u_{i}^{\otimes m_{i}-1} \otimes \cdots \otimes u_{p}^{\otimes m_{p}} \otimes u_{i}(x)\right) \\
& +Y-J \otimes \Delta\left(\begin{array}{|l|l|l}
\hline u_{p}^{m m_{p}} & \cdots & u_{1}^{m}
\end{array}\right) \otimes x .
\end{aligned}
$$

We will first assume that $u_{p}>j_{s+1}$.

What we now have to compute is $\partial_{C} \eta_{q}(Y)+\eta_{q-1} \partial_{C}(Y)$, and show that it equals $Y-\alpha_{q} \theta_{q}(Y)$. Let us first look at

$$
(-1)^{q+1} \partial_{C} \alpha_{0}^{\prime} \theta_{0}^{\prime}\left(J \otimes \Delta\left(\begin{array}{|l|l|l|}
\hline u_{p}^{m_{p}} & \cdots & u_{1}^{m_{1}} \\
\hline
\end{array}\right) \otimes x\right) .
$$

Since $\alpha_{0}^{\prime}$ is the identity, and $\theta_{0}^{\prime}(J)=(-1)^{(s-1)(n-k+1)} j_{s} \cdots j_{n-k+s} \otimes j_{1} \cdots j_{s-1}$, we see that

$$
\begin{aligned}
& (-1)^{q+1} \partial_{C} \alpha_{0}^{\prime} \theta_{0}^{\prime}\left(J \otimes \Delta\left(\begin{array}{|l|l|l|}
\hline u_{p}^{m_{p}} & \cdots & u_{1}^{m_{1}} \\
\hline
\end{array}\right) \otimes x\right) \\
& =J \otimes \Delta\left(\begin{array}{|l|l|l}
\hline u_{p}^{m m_{p}} & \cdots & u_{1}^{m_{1}}
\end{array}\right) \otimes x-(-1)^{\sigma} j_{s} \cdots j_{n-k+s} \\
& \otimes j_{1} \cdots j_{s-1} \Delta\left(\begin{array}{|l|l|l|}
\hline u_{p}^{m m_{p}} & \cdots & u_{1}^{m_{1}} \\
\hline
\end{array}\right) \otimes x \\
& +(-1)^{q+1} \sum_{i=1}^{p}(-1)^{\sigma} j_{s} \cdots j_{n-k+s} \otimes j_{1} \cdots j_{s-1} \\
& \otimes \Delta\left(\begin{array}{|l|l|l|l|l|}
\hline u_{p}^{m_{p}} & \cdots & u_{i}^{m_{i}-1} & \cdots & u_{1}^{m_{1}} \\
\hline
\end{array}\right) \otimes u_{i}(x)
\end{aligned}
$$

(where we have set $\sigma=(s-1)(n-k+1)$ ). Many terms in the boundary disappear due to the fact that $D_{2} \rightarrow D_{1} \otimes D_{1} \rightarrow \Lambda^{2}$ is zero. In any event, we see that adding $(-1)^{q+1} \partial_{C} \alpha_{0}^{\prime} \theta_{0}^{\prime}\left(J \otimes \Delta\left(\begin{array}{|l|l|l|}\hline u_{p}^{m_{p}} & \cdots & u_{1}^{m_{1}}\end{array}\right) \otimes x\right)$ to our expression for $\eta_{q-1} \partial_{C}(Y)$ gives us

$$
\begin{aligned}
& Y-(-1)^{\sigma} j_{s} \cdots j_{n-k+s} \otimes j_{1} \cdots j_{s-1} \Delta\left(\begin{array}{|l|l|l}
\hline u_{p}^{m_{p}} & \cdots & u_{1}^{m_{1}}
\end{array}\right) \otimes x \\
& +(-1)^{q+1} \sum_{i=1}^{p}(-1)^{\sigma} j_{s} \cdots j_{n-k+s} \otimes j_{1} \cdots j_{s-1} \\
& \otimes \Delta\left(\begin{array}{|l|l|l|}
\hline u_{p}^{m_{p}} & \cdots u_{i}^{m_{i}-1} \cdots & u_{1}^{m_{1}}
\end{array}\right) u_{i}(x) \\
& +(-1)^{q} \sum_{i=1}^{p} \eta_{q-1}\left(J u_{i} \otimes u_{1}^{\otimes m_{1}} \otimes \cdots \otimes u_{i-1}^{\otimes m_{i-1}} \otimes u_{i}^{\otimes m_{i}-1} \otimes \cdots \otimes u_{p}^{\otimes m_{p}} \otimes x\right) \\
& +\sum_{i=1}^{p} \eta_{q-1}\left(J \otimes u_{1}^{\otimes m_{1}} \otimes \cdots \otimes u_{i}^{\otimes m_{i}-1} \otimes \cdots \otimes u_{p}^{\otimes m_{p}} \otimes u_{i}(x)\right) .
\end{aligned}
$$

For maximum coverage, we will consider the case that $j_{s} \leq u_{p}$ (for otherwise, $\left.\theta_{q}(Y)=0\right)$. After handling this case, we will indicate what must be added and subtracted in the event that $u_{p}<\cdots<u_{i}<j_{s} \leq u_{i-1}<\cdots<u_{1}$. 
For $l>0$, we have

$$
\begin{aligned}
& (-1)^{q-l+1} \partial_{C} \alpha_{l}^{\prime} \theta_{l}^{\prime}\left(J \otimes \Delta\left(\begin{array}{|l|l|l}
\hline u_{p}^{m m_{p}} & \cdots & u_{1}^{m_{1}} \\
\hline
\end{array}\right) \otimes x\right) \\
& =(-1)^{q-l+1} \sum_{\mathbf{n}} \partial_{C} \alpha_{i}^{\prime} \theta_{i}^{\prime}\left(J \otimes \Delta\left(\begin{array}{|l|l|l}
\hline u_{p}^{n_{p}} & \cdots & u_{1}^{n_{1}} \\
\hline
\end{array}\right)\right. \\
& \left.\otimes \Delta\left(\begin{array}{|l|l|l}
\hline u_{p}^{m_{p}-n_{p}} & \cdots & u_{1}^{m_{1}-n_{1}} \\
\hline
\end{array}\right) \otimes x\right) \\
& =(-1)^{q-l+1} \sum_{\mathbf{n}} \partial_{C}\left((-1)^{\sigma+(s-1) l} \alpha_{i}^{\prime}\left(\begin{array}{cc|c|c|c|}
\hline j_{s} & u_{p}^{n_{p}} & \cdots & u_{1}^{n_{1}} \\
\hline \vdots & & & \\
\cline { 1 - 1 } j_{n-k+s} & & &
\end{array}\right)\right. \\
& \left.\otimes j_{1} \cdots j_{s-1} \otimes \Delta\left(\begin{array}{|l|l|l}
\hline u_{p}^{m_{p}-n_{p}} & \cdots & u_{1}^{m_{1}-n_{1}} \\
\hline
\end{array}\right) \otimes x\right)
\end{aligned}
$$

where $\mathbf{n}=\left(n_{1}, \ldots, n_{p}\right)$ runs through all $p$-tuples of non-negative integers with $n_{1}+\cdots+n_{p}=l$. We see that

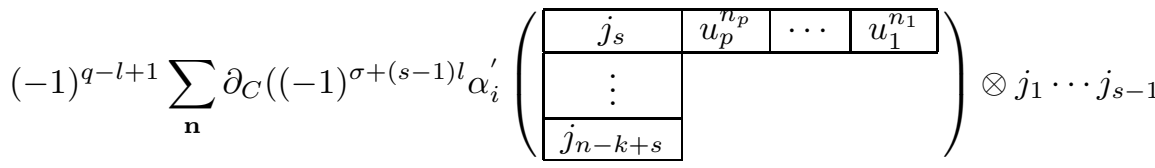

$$
\begin{aligned}
& \left.\otimes \Delta\left(\begin{array}{|l|l|l|}
\hline u_{p}^{m_{p}-n_{p}} & \cdots & u_{1}^{m_{1}-n_{1}} \\
\hline
\end{array}\right) \otimes x\right) \\
& =(\text { up to sign }) \\
& (a)_{l} \quad(-1)^{q-l+1} \sum_{\mathbf{n}} \alpha_{i}^{\prime} \theta_{l}^{\prime}\left(J \otimes u_{1}^{\otimes n_{1}} \otimes \cdots \otimes u_{p}^{\otimes n_{p}}\right) \\
& \otimes \Delta\left(\begin{array}{|l|l|l}
\hline u_{p}^{m_{p}-n_{p}} & \cdots & u_{1}^{m_{1}-n_{1}} \\
\hline
\end{array}\right)(x) \\
& (b)_{l} \quad \pm \sum_{\mathbf{n}} j_{s} \cdots j_{n-k+s} \otimes \Delta\left(\begin{array}{|l|l|l|}
\hline u_{p}^{n_{p}} & \cdots & u_{1}^{n_{1}} \\
\hline
\end{array}\right) \\
& \otimes j_{1} \cdots j_{s-1} \Delta\left(\begin{array}{|l|l|l}
\hline u_{p}^{m_{p}-n_{p}} & \cdots & u_{1}^{m_{1}-n_{1}} \\
\hline
\end{array}\right) \otimes x \\
& (c)_{l} \pm \sum_{\mathbf{n}} \sum_{\lambda} u_{\lambda} j_{s+1} \cdots j_{n-k+s} \otimes \Delta\left(\begin{array}{ll|l|l|l|l|l|}
j_{s} & u_{p}^{n_{p}} & \cdots & u_{\lambda}^{n_{\lambda}-1} & \cdots & u_{1}^{n_{1}} \\
\hline
\end{array}\right) \\
& \otimes j_{1} \cdots j_{s-1} \Delta\left(\begin{array}{|l|l|l|}
\hline u_{p}^{m_{p}-n_{p}} & \cdots & u_{1}^{m_{1}-n_{1}} \\
\hline
\end{array}\right) \otimes x \\
& (d)_{l} \quad \pm \sum_{\mathbf{n}} j_{s} \cdots j_{n-k+s} \otimes \Delta\left(\begin{array}{|l|l|l}
\hline u_{p}^{n_{p}} & \cdots & u_{1}^{n_{1}} \\
\hline & j_{1} \cdots j_{s-1}
\end{array}\right. \\
& \otimes \Delta\left(\begin{array}{|l|l|l}
\hline u_{p}^{m_{p}-n_{p}} & \cdots & u_{1}^{m_{1}-n_{1}} \\
\hline
\end{array}\right) \otimes x \\
& (e)_{l} \quad \pm \sum_{\mathbf{n}} \sum_{\lambda} u_{\lambda} j_{s+1} \cdots j_{n-k+s} \\
& \otimes \Delta\left(\begin{array}{|l|l|l|l|l|l|}
\hline j_{s} & u_{p}^{n_{p}} & \cdots & u_{\lambda}^{n_{\lambda}-1} & \cdots & u_{1}^{n_{1}} \\
\hline
\end{array} j_{1} \cdots j_{s-1}\right. \\
& \otimes \Delta\left(\begin{array}{|l|l|l}
\hline u_{p}^{m_{p}-n_{p}} & \cdots & u_{1}^{m_{1}-n_{1}} \\
\hline
\end{array}\right) \otimes x \text {. }
\end{aligned}
$$


A simple calculation shows that

$$
\alpha_{l}^{\prime} \theta_{l}^{\prime}\left(J \otimes \Delta\left(\begin{array}{|l|l|l|l|l|}
\hline u_{p}^{m_{p}} & \cdots & u_{i}^{m_{i}-1} & \cdots & u_{1}^{m_{1}} \\
\hline
\end{array}\right) \otimes u_{i}(x)\right)
$$

cancels $(a)_{l}$, and that $(d)_{l+1}$ cancels $(b)_{l}$. To handle the next cancellations, let us rewrite the term $(e)_{l}$ as

$$
\begin{aligned}
& \left(e_{1}\right)_{l} \quad \sum_{\mathbf{n}} \sum_{\lambda} u_{\lambda} j_{s+1} \cdots j_{n-k+s} \otimes \Delta\left(\begin{array}{|l|l|l|l|l|}
\hline u_{p}^{n_{p}} & \cdots & u_{\lambda}^{n_{\lambda}-1} & \cdots & u_{1}^{n_{1}} \\
\hline
\end{array}\right) \\
& \otimes j_{1} \cdots j_{s-1} j_{s} \otimes \Delta\left(\begin{array}{|l|l|l}
\hline u_{p}^{m_{p}-n_{p}} & \cdots & u_{1}^{m_{1}-n_{1}} \\
\hline
\end{array}\right) \otimes x \\
& \left(e_{2}\right)_{l}+\sum_{\mathbf{n}} \sum_{\lambda} u_{\lambda} j_{s+1} \cdots j_{n-k+s} \otimes \Delta\left(\begin{array}{|l|l|l|l|l|}
\hline j_{s} & u_{p}^{n_{p}} \cdots & u_{\lambda}^{n_{\lambda}-1} & u_{\mu}^{n_{\mu}-1} \cdots & u_{1}^{n_{1}} \\
\hline
\end{array}\right) \\
& \otimes u_{\mu} j_{1} \cdots j_{s-1} j_{s} \otimes \Delta\left(\begin{array}{|l|l|l}
\hline u_{p}^{m_{p}-n_{p}} & \cdots & u_{1}^{m_{1}-n_{1}} \\
\hline
\end{array}\right) \otimes x .
\end{aligned}
$$

Then we see that $\left(e_{2}\right)_{l+1}$ cancels $(c)_{l}$. Finally, let us evaluate the terms

$$
(-1)^{q} \sum_{i=1}^{p} \eta_{q-1}\left(J u_{i} \otimes u_{1}^{\otimes m_{1}} \otimes \cdots \otimes u_{i}^{\otimes m_{i}-1} \otimes \cdots \otimes u_{p}^{\otimes m_{p}} \otimes x\right)
$$

that remain from $\eta_{q-1} \partial_{C}(Y)$. Again we apply $\alpha_{l}^{\prime} \theta_{l}^{\prime}$ to each of the terms

$$
J u_{i} \otimes u_{1}^{\otimes m_{1}} \otimes \cdots \otimes u_{i}^{\otimes m_{i}-1} \otimes \cdots \otimes u_{p}^{\otimes m_{p}} \otimes x
$$

and add them to the terms that we have left. At this point that is simply $\left(e_{1}\right)_{l}$. We get

$$
\begin{aligned}
& \alpha_{i}^{\prime} \theta_{i}^{\prime}\left(J u _ { i } \otimes \Delta \left(\begin{array}{|l|l|l|l|l}
\hline u_{p}^{m_{p}} & \cdots & u_{i}^{m_{i}-1} & \cdots & u_{1}^{m_{1}} \\
\hline
\end{array}\right.\right. \\
& =\sum_{\mathbf{n}} \alpha_{i}^{\prime} \theta_{i}^{\prime}\left(J u_{i} \otimes \Delta\left(\begin{array}{|l|l|l}
\hline u_{p}^{n_{p}} & \cdots & u_{1}^{n_{1}} \\
\hline
\end{array}\right)\right. \\
& \left.\otimes \Delta\left(\begin{array}{l|l|l|l|l}
\hline u_{p}^{m_{p}-n_{p}} & \cdots & u_{i}^{m_{i}-n_{i}-1} & \cdots & u_{1}^{m_{1}-n_{1}} \\
\hline
\end{array}\right) \otimes x\right) \\
& =\sum_{\mathbf{n}} u_{i} j_{s+1} \cdots j_{n-k+s} \otimes \Delta\left(\begin{array}{|l|l|l|}
\hline u_{p}^{n_{p}} & \cdots & u_{1}^{n_{1}} \\
\hline
\end{array}\right) \otimes j_{1} \cdots j_{s} \\
& \otimes \Delta\left(\begin{array}{|l|l|l|l|l}
\hline u_{p}^{m_{p}-n_{p}} & \cdots & u_{i}^{m_{i}-n_{i}-1} & \cdots & u_{1}^{m_{1}-n_{1}} \\
\hline
\end{array}\right) \otimes x \\
& +\sum_{\mathbf{n}} u_{\lambda} u_{i} j_{s+2} \cdots j_{n-k+s} \otimes \Delta\left(\begin{array}{|l|l|l|l|l|}
\hline u_{p}^{n_{p}} & \cdots & u_{\lambda}^{n_{\lambda}-1} & \cdots & u_{1}^{n_{1}} \\
\hline
\end{array}\right) \otimes j_{1} \cdots j_{s} \\
& \otimes \Delta\left(\begin{array}{|l|l|l|l|l}
\hline u_{p}^{m_{p}-n_{p}} & \cdots & u_{i}^{m_{i}-n_{i}-1} & \cdots & u_{1}^{m_{1}-n_{1}} \\
\hline
\end{array}\right) \otimes x .
\end{aligned}
$$

For the usual reasons, the terms

$$
\begin{aligned}
\sum_{\mathbf{n}} u_{\lambda} u_{i} j_{s+2} \cdots j_{n-k+s} & \otimes \Delta\left(\begin{array}{|l|l|l|l|l|}
\hline u_{p}^{n_{p}} & \cdots & u_{\lambda}^{n_{\lambda}-1} & \cdots & u_{1}^{n_{1}}
\end{array}\right) \otimes j_{1} \cdots j_{s} \\
& \otimes \Delta\left(\begin{array}{|l|l|l|l|l}
u_{p}^{m_{p}-n_{p}} & \cdots & u_{i}^{m_{i}-n_{i}-1} & \cdots & u_{1}^{m_{1}-n_{1}} \\
\hline
\end{array}\right.
\end{aligned}
$$


add up to zero, and the term above that clearly cancels the term $\left(e_{1}\right)_{l+1}$, and it is easy to complete the steps to show that we have the desired result. In particular, we see that the term $(a)_{q}$ does not get cancelled, and that is why the sum we are left with is $Y-\alpha_{q} \theta_{q}(Y)$.

All this was done under the hypothesis that $j_{s} \leq u_{p}$. However, just as we did in the previous lemma, we simply have to keep track of what we add and subtract from the terms above under the assumption that $u_{p}<\cdots<u_{i}<j_{s} \leq u_{i-1}<\cdots<u_{1}$, to get the result in general. For example, if we had $i=p$, we would have to study the terms above under the restriction that in our sequences $\mathbf{n}$, the entry $n_{p}=0$. But this presents no new difficulties.

Now we can state and prove the main theorem.

Theorem 4.3. The map $\eta$ defined above is a homotopy of the identity map on $\mathbf{C}(k ; f)$ with the map $\alpha \theta$. That is, for all $q \geq-1$, we have

$$
\partial_{C} \eta_{q}+\eta_{q-1} \partial_{C}=1-\alpha_{q} \theta_{q}
$$

Proof. It is easy to verify this in the lower dimensions: for $q=-1$, we get zero on both sides. For $q=0$ we take $J \otimes x \in \Lambda^{n-k+s} G^{*} \otimes \Lambda^{n+s} F$ and check that

$$
\begin{aligned}
& \partial_{C}\left(-(-1)^{(s-1)(n-k+1)} j_{s} \cdots j_{n-k+s} \otimes j_{1} \cdots j_{s-1} \otimes x\right) \\
= & -(-1)^{(s-1)(n-k+1)} j_{s} \cdots j_{n-k+s} \otimes j_{1} \cdots j_{s-1}(x)+J \otimes x .
\end{aligned}
$$

In fact, the signs have been rigged so that this does check.

In the general case, we consider the element $Y=J \otimes U_{1} \otimes \cdots \otimes U_{t} \otimes U_{t+1} \otimes$ $u_{1}^{\otimes m_{1}} \otimes \cdots \otimes u_{p}^{\otimes m_{p}} \otimes x$ with $U_{t+1}=v \wedge W$, and $v, u_{1}, \ldots, u_{p}$ all of degree one, as usual, with $u_{1}>\cdots>u_{p}$. We assume that $t$ is some integer lying between 0 and $q-1$, and we set

$$
\begin{aligned}
B(Y) & =J \otimes U_{1} \otimes \cdots \otimes U_{t} v \otimes W \otimes u_{1}^{\otimes m_{1}} \otimes \cdots \otimes u_{p}^{\otimes m_{p}} \otimes x, \\
E(Y) & =J \otimes U_{1} \otimes \cdots \otimes U_{t} \otimes v \otimes u_{1}^{\otimes m_{1}} \otimes \cdots \otimes u_{p}^{\otimes m_{p}} \otimes W(x), \\
\Gamma(Y) & =B(Y)+(-1)^{\sigma} E(Y)
\end{aligned}
$$

with $\sigma=r(q-t-1)$, where degree $(w)=r$. Furthermore, we assume that we have established the fact that $\eta_{i}$ is a homotopy of desired type for $i<q$, and that $\eta_{q}$ is also such on elements of type $Y$ in which the degree of $U_{t+1}$ is less than or equal to $r$. We want to establish the homotopy identity for the element $Y$ whose $(t+1)$ th term is of degree $r+1$. We see immediately, since $\theta_{q}(Y)=\theta_{q}(\Gamma(Y))$, that we have to establish

$$
\partial_{C} \eta_{q}(Y-\Gamma(Y))+\eta_{q-1} \partial_{C}(Y-\Gamma(Y))=Y-\Gamma(Y)
$$

Using a by now familiar type of argument, we see that

$$
\begin{gathered}
\eta_{q-1} \partial_{C}(Y) \\
=(-1)^{q-t-1} \sum_{i=1}^{p} \eta_{q-1}\left(J \otimes U_{1} \otimes \cdots \otimes U_{t} \otimes v W u_{i} \otimes u_{1}^{\otimes m_{1}}\right. \\
\left.\otimes \cdots \otimes u_{i}^{\otimes m_{i}-1} \otimes \cdots \otimes u_{p}^{\otimes m_{p}} \otimes x\right) \\
+\sum_{i=1}^{p} \eta_{q-1}\left(J \otimes U_{1} \otimes \cdots \otimes U_{t} \otimes v W \otimes u_{1}^{\otimes m_{1}} \otimes \cdots \otimes u_{i}^{\otimes m_{i}-1}\right. \\
\left.\otimes \cdots \otimes u_{p}^{\otimes m_{p}} \otimes u_{i}(x)\right)
\end{gathered}
$$




$$
\begin{aligned}
& +(-1)^{q-t} \eta_{q-1}\left(J \otimes U_{1} \otimes \cdots \otimes U_{t} v W \otimes u_{1}^{\otimes m_{1}} \otimes \cdots \otimes u_{p}^{\otimes m_{p}} \otimes x\right) \\
& +(-1)^{q-t} \sum_{i=1}^{t} \eta_{q-1}\left(L_{i}\right)+Y-J \otimes U_{1} \otimes \cdots \otimes U_{t} \otimes v W \\
& \otimes \Delta\left(\begin{array}{|l|l|l}
\hline u_{p}^{m_{p}} & \cdots & u_{1}^{m_{1}} \\
\hline
\end{array}\right.
\end{aligned}
$$

where we set

$$
\begin{aligned}
& L_{i}=J \otimes U_{1} \otimes \cdots \otimes U_{i-1} U_{i} \otimes \cdots \otimes U_{t} \otimes v W \otimes u_{1}^{\otimes m_{1}} \otimes \cdots \otimes u_{p}^{\otimes m_{p}} \otimes x \\
& \quad \eta_{q-1} \partial_{C}(B(Y)) \\
& =\quad(-1)^{q-t-1} \sum_{i=1}^{p} \eta_{q-1}\left(J \otimes U_{1} \otimes \cdots \otimes U_{t} v \otimes W u_{i} \otimes u_{1}^{\otimes m_{1}} \otimes \cdots \otimes u_{i}^{\otimes m_{i}-1}\right. \\
& \left.\quad+\cdots \otimes u_{p}^{\otimes m_{p}} \otimes x\right) \\
& \quad+\sum_{i=1}^{p} \eta_{q-1}\left(B \left(J \otimes U_{1} \otimes \cdots \otimes U_{t} \otimes v W \otimes u_{1}^{\otimes m_{1}} \otimes \cdots \otimes u_{i}^{\otimes m_{i}-1}\right.\right. \\
& \quad+(-1)^{q-t} \eta_{q-1}\left(J \otimes U_{1} \otimes \cdots \otimes U_{t} v W \otimes u_{1}^{\otimes m_{1}} \otimes \cdots \otimes u_{p}^{\otimes m_{p}} \otimes x\right) \\
& \quad+(-1)^{q-t} \sum_{i=1}^{t} \eta_{q-1}\left(B\left(L_{i}\right)\right)+B(Y) \\
& \quad-B\left(J \otimes U_{1} \otimes \cdots \otimes U_{t} \otimes v W \otimes \Delta\left(u_{p}^{m}(x)\right)\right) \\
&
\end{aligned}
$$

where by $B(X)$ we mean the obvious, and

$$
\begin{aligned}
& \eta_{q-1} \partial_{C}(E(Y)) \\
= & (-1)^{q-t-1} \sum_{i=1}^{p} \eta_{q-1}\left(J \otimes U_{1} \otimes \cdots \otimes U_{t} \otimes v u_{i} \otimes u_{1}^{\otimes m_{1}} \otimes \cdots \otimes u_{i}^{\otimes m_{i}-1}\right. \\
& \left.\left.\otimes \cdots \otimes u_{p}^{\otimes m_{p}} \otimes W(x)\right)\right) \\
& (-1)^{r} \sum_{i=1}^{p} \eta_{q-1}\left(E \left(J \otimes U_{1} \otimes \cdots \otimes U_{t} \otimes v W \otimes u_{1}^{\otimes m_{1}}\right.\right. \\
& +(-1)^{q-t} \eta_{q-1}\left(J \otimes U_{1} \otimes \cdots \otimes U_{t} v \otimes u_{1}^{\otimes m_{1}} \otimes \cdots \otimes u_{p}^{\otimes m_{p}} \otimes W(x)\right) \\
& +(-1)^{q-t} \sum_{i=1}^{t} \eta_{q-1}\left(E\left(L_{i}\right)\right)+E(Y) \\
& -E\left(J \otimes U_{1} \otimes \cdots \otimes U_{t} \otimes v W \otimes \Delta\left(u_{p}^{m m_{p}} \cdots \cdots u_{i} \cdots u_{1}^{m_{1}}\right) \otimes x\right)
\end{aligned}
$$


where by $E(X)$ we also mean the obvious. Then

$$
\begin{aligned}
& \eta_{q-1} \partial_{C}(Y-\Gamma(Y)) \\
& =\sum_{i=1}^{p} \eta_{q-1}\left(J \otimes U_{1} \otimes \cdots \otimes U_{t} \otimes v W \otimes u_{1}^{\otimes m_{1}} \otimes \cdots \otimes u_{i}^{\otimes m_{i}-1}\right. \\
& \left.\otimes \cdots \otimes u_{p}^{\otimes m_{p}} \otimes u_{i}(x)\right) \\
& -\sum_{i=1}^{p} \eta_{q-1}\left(\Gamma \left(J \otimes U_{1} \otimes \cdots \otimes U_{t} \otimes v W \otimes u_{1}^{\otimes m_{1}} \otimes \cdots \otimes u_{i}^{\otimes m_{i}-1}\right.\right. \\
& \left.\left.\otimes \cdots \otimes u_{p}^{\otimes m_{p}} \otimes u_{i}(x)\right)\right) \\
& +(-1)^{q-t-1} \sum_{i=1}^{p} \eta_{q-1}\left(J \otimes U_{1} \otimes \cdots \otimes U_{t} \otimes v W u_{i} \otimes u_{1}^{\otimes m_{1}} \otimes \cdots \otimes u_{i}^{\otimes m_{i}-1}\right. \\
& \left.\otimes \cdots \otimes u_{p}^{\otimes m_{p}} \otimes x\right) \\
& -(-1)^{q-t-1} \sum_{i=1}^{p} \eta_{q-1}\left(J \otimes U_{1} \otimes \cdots \otimes U_{t} v \otimes W u_{i} \otimes u_{1}^{\otimes m_{1}} \otimes \cdots \otimes u_{i}^{\otimes m_{i}-1}\right. \\
& \left.\otimes \cdots \otimes u_{p}^{\otimes m_{p}} \otimes x\right) \\
& -(-1)^{\sigma+q-t-1} \sum_{i=1}^{p} \eta_{q-1}\left(J \otimes U_{1} \otimes \cdots \otimes U_{t} \otimes v u_{i} \otimes u_{1}^{\otimes m_{1}} \otimes \cdots \otimes u_{i}^{\otimes m_{i}-1}\right. \\
& \left.\otimes \cdots \otimes u_{p}^{\otimes m_{p}} \otimes W(x)\right) \\
& -(-1)^{\sigma+q-t} \eta_{q-1}\left(J \otimes U_{1} \otimes \cdots \otimes U_{t} v \otimes u_{1}^{\otimes m_{1}}\right. \\
& \left.\otimes \cdots \otimes u_{p}^{\otimes m_{p}} \otimes W(x)\right) \\
& +(-1)^{q-t} \sum_{i=1}^{t} \eta_{q-1}\left(L_{i}-\Gamma\left(L_{i}\right)\right)+(Y-\Gamma(Y)) \\
& -\left(J \otimes U_{1} \otimes \cdots \otimes U_{t} \otimes v W \otimes \Delta\left(\begin{array}{|l|l|l|}
\hline u_{p}^{m m_{p}} & \cdots & u_{1}^{m_{1}} \\
\hline
\end{array}\right) \otimes x\right. \\
& \left.-\Gamma\left(J \otimes U_{1} \otimes \cdots \otimes U_{t} \otimes v W \otimes \Delta\left(\begin{array}{|l|l|l|}
\hline u_{p}^{m_{p}} & \cdots & u_{1}^{m_{1}} \\
\hline
\end{array}\right) \otimes x\right)\right) .
\end{aligned}
$$

The first two rows are, by the definition of our homotopy, equal to

$$
\begin{aligned}
\sum_{i=1}^{p} \sum_{\rho \geq 0} \sum_{|\mathbf{n}|=\rho}(-1)^{(r-1) \rho} Z \otimes \Delta\left(\begin{array}{|l|l|l|l|l}
\hline u_{p}^{n_{p}} & \cdots & u_{1}^{n_{1}} & v
\end{array}\right) \otimes W \\
\qquad \Delta\left(\begin{array}{ll|l|l|l|}
\hline u_{p}^{m_{p}-n_{p}} & \cdots & u_{i}^{m_{i}-n_{i}-1} & \cdots & u_{1}^{m_{1}-n_{1}} \\
\hline
\end{array}\right.
\end{aligned}
$$

where we have set

$$
Z=J \otimes U_{1} \otimes \cdots \otimes U_{t}
$$

and $|\mathbf{n}|=n_{1}+\ldots+n_{p}$. 
The terms $(-1)^{q-t} \sum_{i=1}^{t} \eta_{q-1}\left(L_{i}-\Gamma\left(L_{i}\right)\right)$ are also easy to write down; they are simply

$$
\begin{aligned}
& (-1)^{q-t} \sum_{i=1}^{t} \sum_{\rho \geq 0} \sum_{|\mathbf{n}|=\rho}(-1)^{(r-1) \rho} J \otimes U_{1} \otimes \cdots \otimes U_{i-1} U_{i} \otimes \cdots \otimes U_{t} \\
& \otimes \Delta\left(\begin{array}{|l|l|l|l|}
\hline u_{p}^{n_{p}} & \cdots & u_{1}^{n_{1}} & v \\
\hline
\end{array}\right) \otimes W \otimes \Delta\left(\begin{array}{|l|l|l|}
\hline u_{p}^{m_{p}-n_{p}} & \cdots & u_{1}^{m_{1}-n_{1}} \\
\hline
\end{array} \otimes x\right) .
\end{aligned}
$$

Thus all of our terms but the following have been converted to "non- $\eta$ " terms, and we have to examine their sum:

$$
\begin{gathered}
(-1)^{q-t-1} \sum_{i=1}^{p} \eta_{q-1}\left(J \otimes U_{1} \otimes \cdots \otimes U_{t} \otimes v W u_{i} \otimes u_{1}^{\otimes m_{1}}\right. \\
\left.\otimes \cdots \otimes u_{i}^{\otimes m_{i}-1} \otimes \cdots \otimes u_{p}^{\otimes m_{p}} \otimes x\right) \\
-(-1)^{q-t-1} \sum_{i=1}^{p} \eta_{q-1}\left(J \otimes U_{1} \otimes \cdots \otimes U_{t} v \otimes W u_{i} \otimes u_{1}^{\otimes m_{1}}\right. \\
\left.\otimes \cdots \otimes u_{i}^{\otimes m_{i}-1} \otimes \cdots \otimes u_{p}^{\otimes m_{p}} \otimes x\right) \\
-(-1)^{\sigma+q-t-1} \sum_{i=1}^{p} \eta_{q-1}\left(J \otimes U_{1} \otimes \cdots \otimes U_{t} \otimes v u_{i} \otimes u_{1}^{\otimes m_{1}}\right. \\
\left.\otimes \cdots \otimes u_{i}^{\otimes m_{i}-1} \otimes \cdots \otimes u_{p}^{\otimes m_{p}} \otimes W(x)\right) \\
-(-1)^{\sigma+q-t} \eta_{q-1}\left(J \otimes U_{1} \otimes \cdots \otimes U_{t} v \otimes u_{1}^{\otimes m_{1}} \otimes \cdots \otimes u_{p}^{\otimes m_{p}} \otimes W(x)\right) .
\end{gathered}
$$

There are two possibilities we have to consider: $u_{i}<v$ and $u_{i}>v$. (The case $u_{i}=v$ is easy to dispose of: two of the four summands above are zero, and the other two are very easy to handle.) As usual, we will make the assumption that $v>u_{i}$ for all $i$, and remark as to what happens to various terms that we have to add and/or subtract in the other cases.

Since $v W$ is a basis element, $v$ is less than all the factors of $W$, so $u_{i} v W$ is a basis element and we have

$$
\begin{aligned}
& (-1)^{q-t-1} \sum_{i=1}^{p} \eta_{q-1}\left(J \otimes U_{1} \otimes \cdots \otimes U_{t} \otimes v W u_{i} \otimes u_{1}^{\otimes m_{1}}\right. \\
& \left.\otimes \cdots \otimes u_{i}^{\otimes m_{i}-1} \otimes \cdots \otimes u_{p}^{\otimes m_{p}} \otimes x\right) \\
& =(-1)^{r+q-t} \sum_{i=1}^{p} \eta_{q-1}\left(J \otimes U_{1} \otimes \cdots \otimes U_{t} u_{i} \otimes v W \otimes u_{1}^{\otimes m_{1}} \otimes \cdots \otimes u_{i}^{\otimes m_{i}-1}\right. \\
& \left.\otimes \cdots \otimes u_{p}^{\otimes m_{p}} \otimes x\right) \\
& +(-1)^{r+q-t+(r+1)(q-t-2)} \eta_{q-1}\left(J \otimes U_{1} \otimes \cdots \otimes U_{t} \otimes u_{1}^{\otimes m_{1}}\right. \\
& \left.\otimes \cdots \otimes u_{p}^{\otimes m_{p}} \otimes v W(x)\right) \\
& +(-1)^{r-1} \sum_{i=1}^{p} \sum_{\rho \geq 0} \sum_{|\mathbf{n}|=\rho}(-1)^{r \rho} Z \otimes \Delta\left(\begin{array}{|l|l|l|l|}
\hline u_{p}^{n_{p}} & \cdots & u_{1}^{n_{1}} & u_{i} \\
\hline
\end{array}\right) \otimes v W \\
& \otimes \Delta\left(\begin{array}{|l|l|l|l|l}
\hline u_{p}^{m_{p}-n_{p}} & \cdots & u_{i}^{m_{i}-n_{i}-1} & \cdots & u_{1}^{m_{1}-n_{1}} \\
\hline
\end{array}\right) \otimes x .
\end{aligned}
$$

(The penultimate term has no summation in it because the terms of the same sort coming from $i>1$ all vanish.) 
Next we see that

$$
\begin{aligned}
& (-1)^{\sigma+q-t-1} \sum_{i=1}^{p} \eta_{q-1}\left(J \otimes U_{1} \otimes \cdots \otimes U_{t} \otimes v u_{i} \otimes u_{1}^{\otimes m_{1}} \otimes \cdots \otimes u_{i}^{\otimes m_{i}-1}\right. \\
& \left.\otimes \cdots \otimes u_{p}^{\otimes m_{p}} \otimes W(x)\right) \\
& =(-1)^{\sigma+q-t} \sum_{i=1}^{p} \eta_{q-1}\left(J \otimes U_{1} \otimes \cdots \otimes U_{t} u_{i} \otimes v \otimes u_{1}^{\otimes m_{1}}\right. \\
& \left.\otimes \cdots \otimes u_{i}^{\otimes m_{i}-1} \otimes \cdots \otimes u_{p}^{\otimes m_{p}} \otimes W(x)\right) \\
& +(-1)^{\sigma-2} \eta_{q-1}\left(J \otimes U_{1} \otimes \cdots \otimes U_{t} \otimes u_{1}^{\otimes m_{1}} \otimes \cdots \otimes u_{p}^{\otimes m_{p}} \otimes v W(x)\right) \\
& +(-1)^{\sigma+q-t} \sum_{i=1}^{p} \sum_{\rho \geq 0} \sum_{|\mathbf{n}|=\rho} Z \otimes \Delta\left(\begin{array}{|l|l|l|l|}
\hline u_{p}^{n_{p}} & \cdots & u_{1}^{n_{1}} & u_{i} \\
\hline
\end{array}\right) \otimes v \\
& \otimes \Delta\left(\begin{array}{|l|l|l|l|l}
\hline u_{p}^{m_{p}-n_{p}} & \cdots & u_{i}^{m_{i}-n_{i}-1} & \cdots & u_{1}^{m_{1}-n_{1}} \\
\hline
\end{array}\right) \otimes W(x) .
\end{aligned}
$$

When we collect our terms we have

$$
\begin{aligned}
& (-1)^{r+q-t} \sum_{i=1}^{p} \eta_{q-1}\left(J \otimes U_{1} \otimes \cdots \otimes U_{t} u_{i} \otimes v W \otimes u_{1}^{\otimes m_{1}}\right. \\
& \left.\otimes \cdots \otimes u_{i}^{\otimes m_{i}-1} \otimes \cdots \otimes u_{p}^{\otimes m_{p}} \otimes x\right) \\
& +(-1)^{\sigma+q-t} \sum_{i=1}^{p} \eta_{q-1}\left(J \otimes U_{1} \otimes \cdots \otimes U_{t} u_{i} \otimes v \otimes u_{1}^{\otimes m_{1}}\right. \\
& \left.\otimes \cdots \otimes u_{i}^{\otimes m_{i}-1} \otimes \cdots \otimes u_{p}^{\otimes m_{p}} \otimes W(x)\right) \\
& -(-1)^{q-t-1} \sum_{i=1}^{p} \eta_{q-1}\left(J \otimes U_{1} \otimes \cdots \otimes U_{t} v \otimes W u_{i} \otimes u_{1}^{\otimes m_{1}}\right. \\
& \left.\otimes \cdots \otimes u_{i}^{\otimes m_{i}-1} \otimes \cdots \otimes u_{p}^{\otimes m_{p}} \otimes x\right) \\
& -(-1)^{\sigma+q-t} \sum_{i=1}^{p} \eta_{q-1}\left(J \otimes U_{1} \otimes \cdots \otimes U_{t} v \otimes u_{1}^{\otimes m_{1}} \otimes \cdots \otimes u_{p}^{\otimes m_{p}} \otimes W(x)\right) \\
& +(-1)^{r-1} \sum_{i=1}^{p} \sum_{\rho \geq 0} \sum_{|\mathbf{n}|=\rho}(-1)^{r \rho} Z \otimes \Delta\left(\begin{array}{|l|l|l|l|}
\hline u_{p}^{n_{p}} & \cdots & u_{1}^{n_{1}} & u_{i} \\
\hline
\end{array}\right) \otimes v W \\
& \otimes \Delta\left(\begin{array}{|l|l|l|l|l}
\hline u_{p}^{m_{p}-n_{p}} & \cdots & u_{i}^{m_{i}-n_{i}-1} & \cdots & u_{1}^{m_{1}-n_{1}} \\
\hline
\end{array}\right) \otimes x \\
& +(-1)^{\sigma+q-t} \sum_{i=1}^{p} \sum_{\rho \geq 0} \sum_{|\mathbf{n}|=\rho} Z \otimes \Delta\left(\begin{array}{|l|l|l|l}
u_{p}^{n_{p}} & \cdots & u_{1}^{n_{1}} & u_{i} \\
\hline
\end{array}\right) \otimes v \\
& \otimes \Delta\left(\begin{array}{|l|l|l|l|l|}
\hline u_{p}^{m_{p}-n_{p}} & \cdots & u_{i}^{m_{i}-n_{i}-1} & \cdots & u_{1}^{m_{1}-n_{1}} \\
\hline
\end{array}\right) \otimes W(x) .
\end{aligned}
$$


Next, we see that

$$
\begin{aligned}
& (-1)^{q-t-1} \sum_{i=1}^{p} \eta_{q-1}\left(J \otimes U_{1} \otimes \cdots \otimes U_{t} v \otimes W u_{i} \otimes u_{1}^{\otimes m_{1}}\right. \\
& \left.\otimes \cdots \otimes u_{i}^{\otimes m_{i}-1} \otimes \cdots \otimes u_{p}^{\otimes m_{p}} \otimes x\right) \\
& =(-1)^{q-t-1+r} \sum_{i=1}^{p} \eta_{q-1}\left(J \otimes U_{1} \otimes \cdots \otimes U_{t} v u_{i} \otimes W \otimes u_{1}^{\otimes m_{1}}\right. \\
& \left.\otimes \cdots \otimes u_{i}^{\otimes m_{i}-1} \otimes \cdots \otimes u_{p}^{\otimes m_{p}} \otimes x\right) \\
& +(-1)^{q-t-1+r+r(q-t-2)} \eta_{q-1}\left(J \otimes U_{1} \otimes \cdots \otimes U_{t} v \otimes \otimes u_{1}^{\otimes m_{1}}\right. \\
& \left.\otimes \cdots \otimes u_{p}^{\otimes m_{p}} \otimes W(x)\right) \\
& +(-1)^{r} \sum_{i=1}^{p} \sum_{\rho \geq 0} \sum_{|\mathbf{n}|=\rho}(-1)^{(r-1) \rho} J \otimes U_{1} \otimes \cdots \otimes U_{t} v \\
& \otimes \Delta\left(\begin{array}{|l|l|l|l|}
\hline u_{p}^{n_{p}} & \cdots & u_{1}^{n_{1}} & u_{i} \\
\hline
\end{array}\right) \otimes W \\
& \otimes \Delta\left(\begin{array}{|l|l|l|l|l}
\hline u_{p}^{m_{p}-n_{p}} & \cdots & u_{i}^{m_{i}-n_{i}-1} & \cdots & u_{1}^{m_{1}-n_{1}} \\
\hline
\end{array}\right) \otimes x
\end{aligned}
$$

so that our collected terms now are

$$
\begin{aligned}
& (-1)^{r+q-t} \sum_{i=1}^{p} \eta_{q-1}\left(J \otimes U_{1} \otimes \cdots \otimes U_{t} u_{i} \otimes v W \otimes u_{1}^{\otimes m_{1}} \otimes \cdots \otimes\right. \\
& \left.u_{i}^{\otimes m_{i}-1} \otimes \cdots \otimes u_{p}^{\otimes m_{p}} \otimes x\right) \\
& +(-1)^{\sigma+q-t} \sum_{i=1}^{p} \eta_{q-1}\left(J \otimes U_{1} \otimes \cdots \otimes U_{t} u_{i} \otimes v \otimes u_{1}^{\otimes m_{1}} \otimes \cdots \otimes\right. \\
& \left.u_{i}^{\otimes m_{i}-1} \otimes \cdots \otimes u_{p}^{\otimes m_{p}} \otimes W(x)\right) \\
& -(-1)^{q-t+r} \sum_{i=1}^{p} \eta_{q-1}\left(J \otimes U_{1} \otimes \cdots \otimes U_{t} u_{i} v \otimes W \otimes u_{1}^{\otimes m_{1}} \otimes \cdots \otimes\right. \\
& \left.u_{i}^{\otimes m_{i}-1} \otimes \cdots \otimes u_{p}^{\otimes m_{p}} \otimes x\right) \\
& +(-1)^{r-1} \sum_{i=1}^{p} \sum_{\rho \geq 0} \sum_{|\mathbf{n}|=\rho}(-1)^{r \rho} Z \otimes \Delta\left(\begin{array}{|l|l|l|l|}
\hline u_{p}^{n_{p}} & \cdots & u_{1}^{n_{1}} & u_{i} \\
\hline
\end{array}\right) \otimes v W \\
& \otimes \Delta\left(\begin{array}{|l|l|l|l|l}
\hline u_{p}^{m_{p}-n_{p}} & \cdots & u_{i}^{m_{i}-n_{i}-1} & \cdots & u_{1}^{m_{1}-n_{1}} \\
\hline
\end{array}\right. \\
& +(-1)^{\sigma+q-t} \sum_{i=1}^{p} \sum_{\rho \geq 0} \sum_{|\mathbf{n}|=\rho} Z \otimes \Delta\left(\begin{array}{|l|l|l|l|}
\hline u_{p}^{n_{p}} & \cdots & u_{1}^{n_{1}} & u_{i} \\
\hline
\end{array}\right) \otimes v \\
& \left.\otimes \Delta\left(\begin{array}{|l|l|l|l|l}
\hline u_{p}^{m_{p}-n_{p}} & \cdots & u_{i}^{m_{i}-n_{i}-1} & \cdots & u_{1}^{m_{1}-n_{1}} \\
\hline
\end{array}\right) \otimes W(x)\right) \\
& +(-1)^{r} \sum_{i=1}^{p} \sum_{\rho \geq 0} \sum_{|\mathbf{n}|=\rho}(-1)^{(r-1) \rho} J \otimes U_{1} \otimes \cdots \otimes U_{t} v \\
& \otimes \Delta\left(\begin{array}{|l|l|l|l|}
\hline u_{p}^{n_{p}} & \cdots & u_{1}^{n_{1}} & u_{i} \\
\left.\hline \begin{array}{ll}
\hline \\
m_{p}
\end{array}\right) \otimes W
\end{array}\right. \\
& \otimes \Delta\left(\begin{array}{|l|l|l|l|l|}
\hline u_{p}^{m_{p}-n_{p}} & \cdots & u_{i}^{m_{i}-n_{i}-1} & \cdots & u_{1}^{m_{1}-n_{1}} \\
\hline
\end{array}\right) \otimes x .
\end{aligned}
$$


Finally, we have that the first three rows add up to

$$
\begin{aligned}
& (-1)^{r-1} \sum_{i=1}^{p} \sum_{\rho \geq 0} \sum_{|\mathbf{n}|=\rho}(-1)^{(r-1) \rho} J \otimes U_{1} \otimes \cdots \otimes U_{t} u_{i} \\
& \otimes \Delta\left(\begin{array}{|l|l|l|l|}
\hline u_{p}^{n_{p}} & \cdots & u_{1}^{n_{1}} & v \\
\hline
\end{array}\right) \otimes W \\
& \otimes \Delta\left(\begin{array}{|l|l|l|l|l}
\hline u_{p}^{m_{p}-n_{p}} & \cdots & u_{i}^{m_{i}-n_{i}-1} & \cdots & u_{1}^{m_{1}-n_{1}} \\
\hline
\end{array}\right) \otimes x,
\end{aligned}
$$

so that we end up with our collected terms summing to

$$
\begin{aligned}
& (-1)^{r-1} \sum_{i=1}^{p} \sum_{\rho \geq 0} \sum_{|\mathbf{n}|=\rho}(-1)^{r \rho} Z \otimes \Delta\left(\begin{array}{|l|l|l|l|}
\hline u_{p}^{n_{p}} & \cdots & u_{1}^{n_{1}} & u_{i} \\
\hline
\end{array}\right) \otimes v W \\
& \otimes \Delta\left(\begin{array}{|l|l|l|l|l}
\hline u_{p}^{m_{p}-n_{p}} & \cdots & u_{i}^{m_{i}-n_{i}-1} & \cdots & u_{1}^{m_{1}-n_{1}} \\
\hline
\end{array}\right) \otimes x \\
& +(-1)^{\sigma+q-t} \sum_{i=1}^{p} \sum_{\rho \geq 0} \sum_{|\mathbf{n}|=\rho} Z \otimes \Delta\left(\begin{array}{|l|l|l|l|}
\hline u_{p}^{n_{p}} & \cdots & u_{1}^{n_{1}} & u_{i} \\
\hline
\end{array}\right) \otimes v \\
& \otimes \Delta\left(\begin{array}{l|l|l|l|l}
\hline u_{p}^{m_{p}-n_{p}} & \cdots & u_{i}^{m_{i}-n_{i}-1} & \cdots & u_{1}^{m_{1}-n_{1}} \\
\hline
\end{array}\right) \otimes W(x) \\
& +(-1)^{r} \sum_{i=1}^{p} \sum_{\rho \geq 0} \sum_{|\mathbf{n}|=\rho}(-1)^{(r-1) \rho} J \otimes U_{1} \otimes \cdots \otimes U_{t} v \\
& \otimes \Delta\left(\begin{array}{|l|l|l|l|}
\hline u_{p}^{n_{p}} & \cdots & u_{1}^{n_{1}} & u_{i} \\
\hline
\end{array}\right) \otimes W \\
& \otimes \Delta\left(\begin{array}{|l|l|l|l|l}
u_{p}^{m_{p}-n_{p}} & \cdots & u_{i}^{m_{i}-n_{i}-1} & \cdots & u_{1}^{m_{1}-n_{1}} \\
\hline
\end{array}\right) \otimes x \\
& +(-1)^{r-1} \sum_{i=1}^{p} \sum_{\rho \geq 0} \sum_{|\mathbf{n}|=\rho}(-1)^{(r-1) \rho} J \otimes U_{1} \otimes \cdots \otimes U_{t} u_{i} \\
& \otimes \Delta\left(\begin{array}{|l|l|l|l|}
\hline u_{p}^{n_{p}} & \cdots & u_{1}^{n_{1}} & v \\
\hline
\end{array}\right) \otimes W \\
& \otimes \Delta\left(\begin{array}{|l|l|l|l|l|}
u_{p}^{m_{p}-n_{p}} & \cdots & u_{i}^{m_{i}-n_{i}-1} & \cdots & u_{1}^{m_{1}-n_{1}} \\
\hline
\end{array}\right) \otimes x .
\end{aligned}
$$

All of the above was by way of calculating $\eta_{q-1} \partial_{C}(Y-\Gamma(Y))$. We now have to add the terms of $\partial_{C} \eta_{q}(Y-\Gamma(Y))$ to what we have obtained. But we know that

$$
\begin{array}{r}
\eta_{q}(Y-\Gamma(Y))=\sum_{\rho \geq 0} \sum_{\mathbf{n}}(-1)^{(r-1) \rho} Z \otimes \Delta\left(\begin{array}{|l|l|l|l|}
\hline u_{p}^{n_{p}} & \cdots & u_{1}^{n_{1}} & v
\end{array}\right) \otimes W \\
\qquad \Delta\left(\begin{array}{|l|l|l}
u_{p}^{m_{p}-n_{p}} & \cdots & u_{1}^{m_{1}-n_{1}} \\
\hline
\end{array}\right) \otimes x .
\end{array}
$$

If we take $\partial_{C} \eta_{q}(Y-\Gamma(Y))$, we see that the boundary applied to the $Z$ part of these terms just gives us our terms related to our $L_{i}$ terms above. The boundary term corresponding to the action on $x$ is covered by terms calculated at the outset. The terms

$$
\begin{aligned}
\left(J \otimes U_{1} \otimes \cdots \otimes U_{t}\right. & \otimes v W \otimes \Delta\left(\begin{array}{|l|l|l|l|l|}
\hline u_{p}^{m m_{p}} & \cdots & u_{1}^{m_{1}}
\end{array}\right) \otimes x \\
& -\Gamma\left(J \otimes U _ { 1 } \otimes \cdots \otimes U _ { t } \otimes v W \otimes \Delta \left(\begin{array}{|l|l|l}
\hline u_{p}^{m m_{p}} & \cdots & u_{1}^{m_{1}} \\
\hline
\end{array}\right.\right.
\end{aligned}
$$

that we found earlier are taken care of in part by the $W(x)$ terms we have found just above. The rest of the terms that we have laboriously calculated deal with the interactions of the $v$ with $W$, and with the multiplication of these with their 
immediate neighbors that occur when we apply the boundary. In short, we can check off, term by term, the results of applying the boundary and our collected terms, taking into account a certain amount of internal cancellation (such as the $W(x)$ terms just mentioned).

All of the above was predicated on the assumption that $v>u_{1}$. If we had $u_{1}>v>u_{2}$, say, then the terms involving $\Delta\left(\begin{array}{|l|l|l|l|}\hline u_{p}^{n_{p}} & \cdots & u_{1}^{n_{1}} & v \\ \hline\end{array}\right.$ the requirement that $n_{1}=0$. On the other hand, when we calculated the " $\eta$ " terms of our $\eta_{q-1} \partial_{C}(Y-\Gamma(Y))$, we would have had to keep the $v$ in front (other signs involved in 'rectifying' the product $W u_{1}$ would have cancelled each other out), and we would have had a corresponding gain or loss of our resulting "non- $\eta$ " terms. Thus we have our desired result.

\section{REFERENCES}

[1] K. Akin, D. A. Buchsbaum and J. Weyman. Schur functors and Schur complexes. Advances in Math. 44 (1982), 207-278. MR 84c:20021

[2] D. A. Buchsbaum. A generalized Koszul complex, I. Trans. Amer. Math. Soc. 111 (1964), 183-196. MR 28:3075

[3] D. A. Buchsbaum and D. S. Rim. A generalized Koszul complex, II. Depth and multiplicity. Trans. Amer. Math. Soc. 111 (1964), 197-224. MR 28:3076]

[4] D. A. Buchsbaum and D. S. Rim. A generalized Koszul complex, III. Proc. Amer. Math. Soc. 16 (1965), 555-558. MR 31:1285

[5] D. A. Buchsbaum and D. Eisenbud. Generic free resolutions and a family of generically perfect ideals. Advances in Math. 18 (1975), 245-301. MR 53:391

[6] J. Eagon and D.G. Northcott. Ideals defined by matrices and a certain complex associated with them. Proc. Roy. Soc. London Ser. A 269 (1962), 188-204. MR 26:161

[7] E. Gover. Generalized local complete intersections Thesis, Brandeis University (1970).

[8] S. Kleiman and A. Thorup. A geometric theory of the Buchsbaum-Rim multiplicity. J. Algebra 167 (1994), 168-231. MR 96a:14007

[9] S. Kleiman and A. Thorup. Mixed Buchsbaum-Rim multiplicities. Amer. J. Math. 118 (1996), 529-569. MR 98g:14008

Dipartimento di Scienze, Università “G. D’Annunzio”, Viale Pindaro 42, 65127 Pescara, Italy

E-mail address: gboffi@unich.it

Department of Mathematics, Brandeis University, Waltham, Massachusetts 02254

E-mail address: buchsbau@brandeis.edu 